\author{
Felipe Antonio C. Viana \\ fchegury@mecanica.ufu.br \\ and Valder Steffen, Jr \\ School of Mechanical Engineering, Federal \\ University of Uberlândia \\ Av. João Naves de Ávila 2121 \\ Campus Santa Mônica \\ P.O. Box 593 \\ 38400-902 Uberlândia, MG. Brazil \\ vsteffen@mecanica.ufu.br
}

\section{Multimodal Vibration Damping through Piezoelectric Patches and Optimal Resonant Shunt Circuits}

Piezoelectric elements connected to shunt circuits and bonded to a mechanical structure form a dissipation device that can be designed to add damping to the mechanical system. Due to the piezoelectric effect, part of the vibration energy is transformed into electrical energy that can be conveniently dissipated. Therefore, by using appropriate electrical circuits, it is possible to dissipate strain energy and, as a consequence, vibration is suppressed through the added passive damping. From the electrical point of view, the piezoelectric element behaves like a capacitor in series with a controlled voltage source and the shunt circuit, commonly formed by an RL network, is tuned to dissipate the electrical energy, more efficiently in a given frequency band. It is important to know that large inductances are frequently required, leading to the necessity of using synthetic inductors (obtained from operational amplifiers). From the mechanical point of view, the vibration energy can be attenuated in a single mode, or in multiple modes, according to the design of the damping device and the frequency band of interest. This work is devoted to the study of passive damping systems for single modes or multiple modes, based on piezoelectric patches and resonant shunt circuits. The present contribution discusses the modeling of piezoelectric patches coupled to shunt circuits, where the basics of resonant shunt circuits (series and parallel topologies) are presented. Following, the devices used in passive control (piezoelectric patch and synthetic inductors) are analyzed from the electrical and experimental viewpoints. The modeling of multi-degree-of-freedom mechanical systems, including the effects of the passive damping devices is revisited, and, then a design methodology for the multi-modal case is defined. Also, it is briefly reviewed the optimization method used for design purposes, namely the LifeCycle Model. Finally, experimental results are reported, illustrating the success of using the methodology presented in passive damping applications applied to mechanical and mechatronic systems.

Keywords: Multimodal damping, shunted piezoelectric, resonant shunt circuits

\section{Introduction}

Applications of smart materials are growing and nowadays they cover from military applications to the industry of consumption. Among other applications, the following can be mentioned:

Vibration damping in sport items represents an important issue for a new generation of ski boards, tennis rackets and golf and baseball bats (Figure 1). The goal here is to reduce vibrations in these sport items, increasing the user's comfort and preventing damage.

A group composed by Boeing Company, the University of Maryland, the Massachusetts Institute of Technology, the University of California (Los Angeles) and the U.S. Army Research Office support projects for the development and application of smart materials. For example, the Smart Helicopter Rotor can be mentioned (Figure 2), in which fibers of composite materials and piezoelectric devices are used in the blades of the helicopter rotor to attenuate vibrations and noise and also to improve the aerodynamic performance.

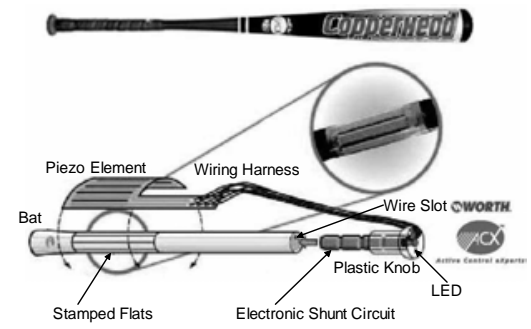

Figure 1. Worth Copperhead ACX Adult Baseball Bat, first commercialized in 1998 (adapted from Akhras, 2000).

Paper accepted May, 2006. Technical Editor: José Roberto de França Arruda.

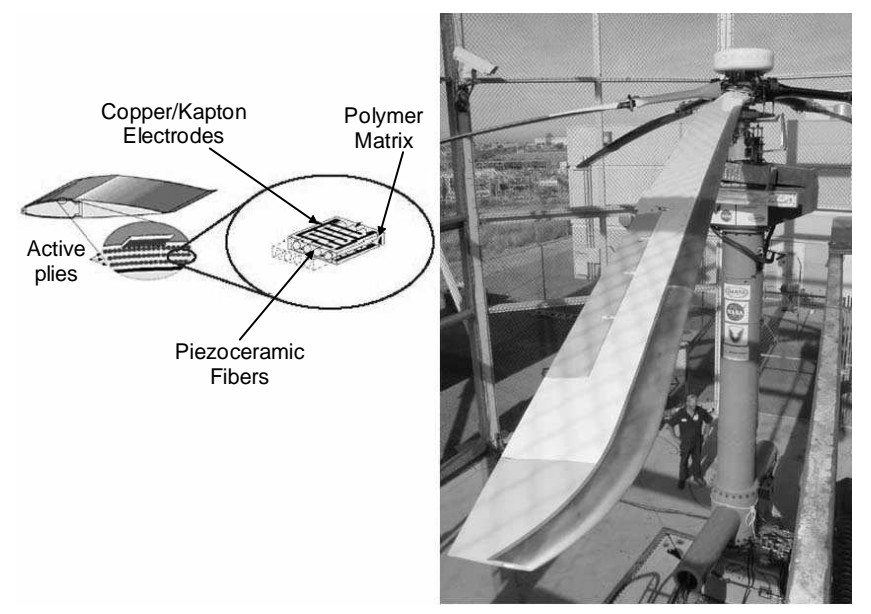

Figure 2. Smart Helicopter Rotor (http://www.boeing.com/news/releases/, July 2004).

Applications related to vibration control may use either passive or active techniques, as well as a combination of both. Actuators, power supplies and control systems characterize the active techniques. In passive techniques, the power supplies and control systems are suppressed and the shape and physical characteristics of smart materials are explored for vibration reduction.

Passive damping, according to Johnson (1995) can be divided into two classes: structural and embedded. The structural damping occurs due the friction of junctions, cable rubbing and material damping. The embedded damping is achieved by adding dissipation mechanisms to the structure, commonly based on one of the following damping techniques: viscoelastic materials, viscous 
devices, magnetic devices and passive piezoelectrics. Table 1 shows a comparison of these approaches.

Due to the piezoelectric effect, a portion of the mechanical energy associated with the vibration can be transformed into electric energy and dissipated conveniently, through a shunt circuit that compounds a mechanism of passive damping. Lesieutre (1998) discusses the four commonly used types of shunt circuits: resistive, resonant, capacitive and switched, as shown in Table 2. The abbreviation PZT (lead-zirconate-titanate) refers to the piezoelectric element. This way, $C_{P Z T}$ is the inherent capacitance of the piezoelectric patch.

Among the shunt circuits, the resonant one deserves a special attention. Compound by an inductor and a resistor, this circuit allows the tuning to any desired frequency, which will have the corresponding vibration amplitudes attenuated. In addition, improvements in the topology of the circuit make possible the simultaneous reduction of more than one vibration mode. From the mechanical point of view, the system as a whole (PZT and resonant shunt circuit) is similar to the dynamic vibration absorber.

A detailed description of the use of shunt circuit and piezoelectric devices is seen in the pioneer work of Hagood and von Flotow (1991). In that work, the expression for the mechanical impedance introduced by the piezoelectric element connected to any type of shunt circuit coupled to mechanical systems is obtained. Two study cases are presented, showing experiments with shunt circuits, both resistive and series resonant types.

Table 1. Primary passive damping mechanisms and correlated information (adapted from Johnson, 1995).

\begin{tabular}{|l|c|c|c|c|}
\cline { 2 - 5 } \multicolumn{1}{c|}{} & \multicolumn{3}{c|}{ Type of damping mechanism } \\
\cline { 2 - 5 } \multicolumn{1}{c|}{} & Viscoelastic materials & Viscous devices & Magnetic devices & Passive piezoelectrics \\
\hline Type of treatment & All & Struts and DVAs ${ }^{*}$ & Struts and DVAs & Strut dampers \\
\hline Temperature sensitivity & High & Moderate & Low & Low \\
\hline Temperature range & Moderate & Moderate & Wide & Wide \\
\hline Loss factor & Moderate & High & Low & Low \\
\hline Frequency range & Wide & Moderate & Moderate & Moderate \\
\hline Weight & Low & Moderate & High & Moderate \\
\hline
\end{tabular}

Table 2. Shunt circuits (adapted from Lesiutre, 1998).

\begin{tabular}{|l|l|l|}
\hline Resistive & Resonant \\
This circuit results a behavior that \\
is similar to viscoelastically- \\
damped systems.
\end{tabular}

A series of papers have been reported about the present topic. $\mathrm{Wu}$ (1996) makes considerations about the behavior of the resonant shunt circuit in the parallel topology. Steffen and Inman (1999) use control techniques and optimization strategies in the design of both active and passive vibration reduction devices, using piezoelectric materials. Steffen et al. (2000) examine the use of passive techniques for vibration reduction combining dynamic vibration absorbers (DVAs) and piezoelectric patches coupled to resonant shunt circuits. In that work, natural optimization techniques were used in the design of the damping system. Park and Inman (2003) discuss the non-idealities of the synthetic inductor used in the shunt circuits and propose an improvement by adding capacitors in parallel to the piezo, aiming at reducing the values required for the inductance.

This work is devoted to the study of passive damping systems for single modes or multiple modes. As compared to previous contributions, this paper presents a complete study concerning resonant shunted piezoelectric, including the analytical and experimental aspects together with the synthetic inductor design. Besides, the study devoted to the multimode case represents a more realistic approach. Previous works considered the multimode case a simple extension of the single mode case. Also, the use of optimization techniques is considered a valuable contribution because no closed solution can be derived for the multimode case. The optimization results were validated experimentally. The present contribution is organized as follows: first, the modeling of piezoelectric patches coupled to shunt circuits, where the basics of resonant shunt circuits (series and parallel) are presented together with a literature review about this topic; following, the devices used in passive control (piezoelectric patch and synthetic inductors Riordan and Antoniou types) are analyzed from both the electrical and experimental viewpoints; then the modeling of multi-degree-offreedom mechanical systems, including the effects of the passive damping mechanism is presented; in the sequence, a new design methodology for the multi-modal case is proposed. Also, the optimization method used for design purposes, namely the LifeCycle Model, is briefly reviewed. Finally, experimental results are reported, illustrating the success of using the methodology presented as applied to passive damping applications of mechanical and mechatronic structures.

\section{Nomenclature}

$\mathbf{A}=$ the matrix of surfaces, perpendicular to the electric field

$A_{i}$ and $A_{j}=$ the transversal section areas according to the vectors $\mathbf{i}$ and $\mathbf{j}\left[\mathrm{m}^{2}\right]$

$\mathbf{B}=$ the diagonal matrix of the lengths of the piezoelectric patch 
$\mathrm{B}_{\mathbf{i}}$ and $\mathrm{B}_{\mathbf{j}}=$ the initial patch lengths according to vectors $\mathbf{i}$ and $\mathbf{j}[m]$

$C_{P Z T}^{T}=$ the inherent capacitance of the piezoelectric patch taken at constant stress (free)

$\mathbf{D}=$ the vector of electrical displacement $\left[{\left.\mathrm{C} / \mathrm{m}^{2}\right]}^{2}\right.$

$\mathbf{E}=$ vector of electric field $[\mathrm{V} / \mathrm{m}]$

$\mathbf{I}=$ the electric current

$K=$ the stiffness of the mechanical system

$K_{\mathbf{j} j}^{E}$ is the stiffness of the piezoelectric patch taken in open circuit

$L=$ the inductance

$\mathrm{PZT}=$ piezoelectric patch

$R=$ the resistance

$\mathbf{S}=$ the vector of material engineering strains (nondimensional)

$\mathrm{S}_{\mathbf{j}}=$ the strain (non-dimensional)

$\mathbf{T}=$ vector of material stresses $\left[\mathrm{N} / \mathrm{m}^{2}\right]$

$\mathrm{T}_{\mathbf{j}}=$ the stress $\left[\mathrm{N} / \mathrm{m}^{2}\right]$

$\mathbf{V}=$ the electric voltage

$\mathbf{Y}=$ the electric admittance

$Z_{\mathbf{i}}^{\text {SHUNT }}(s)=$ the electrical impedance of the shunt circuit $[\Omega]$

$Z_{\mathbf{j} \mathbf{j}}^{S H N T}(s)=$ the mechanical impedance of the passive damping device with the shunt circuit shunt

$\mathbf{d}=$ the piezoelectric material constant relating strain to voltage $[\mathrm{m} / \mathrm{V}]$

$d_{\mathbf{i j}}=$ the piezoelectric material constant relating voltage in ith direction to strain in the $\mathbf{j t h}$ direction

$\mathbf{i}$ and $\mathbf{j}=$ coordinate axes that indicate the direction for electrical and mechanical vectors

$k=$ electromechanical coupling coefficient

$k_{r}=$ the modal stiffness related to the mode $\mathrm{r}$

$m_{r}=$ the modal mass related to the mode $\mathrm{r}$

$\mathbf{s}=$ the matrix of compliance for the material $\left[\mathrm{m}^{2} / \mathrm{N}\right]$

$s=$ the Laplace variable

\section{Greek Symbols}

$\boldsymbol{\varepsilon}=$ the matrix of dielectric constants for the material $\left[\mathrm{C}^{2} /\left(\mathrm{N} \cdot \mathrm{m}^{2}\right)\right]$

$\delta=$ the non-dimensional tuning ratio

$\omega_{0}=$ the electrical resonance frequency $[\mathrm{rad} / \mathrm{s}]$

$\omega_{n}=$ the resonance frequency of the mechanical system $[\mathrm{rad} / \mathrm{s}]$

$\xi=$ the damping factor

$\gamma=$ the non-dimensional frequency

\section{Subscripts}

PZT relative to the piezoelectric patch

\section{Superscripts}

$E \quad$ value taken at constant field (short circuit)

ELECT relative to an electrical parameter

$D \quad$ value taken at constant electrical displacement (open circuit)

$M E C \quad$ relative to a mechanical parameter

RSP pertaining to resonant circuit shunting

$S \quad$ value taken at constant strain (clamped)

OPTM relative to the optimal condition

PARALLEL relative to the parallel circuit topology
SERIES relative to the series circuit topology

SHUNT relative to the shunt circuit

$T \quad$ value taken at constant stress (free)

$t \quad$ transpose of the vector or matrix

\section{Modeling Piezoelectric Patches coupled to Shunt Circuits}

Figure 3 illustrates the case in which a piezoelectric patch is coupled to a shunt circuit and bonded to a flexible structure. Figure 3-(a) shows the complete system and Figure 3-(b) depicts the simplified model for the passive damping device.
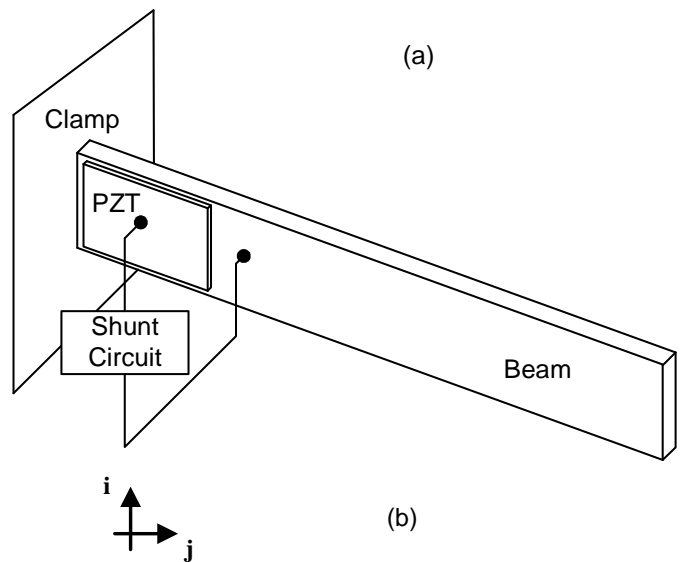

(b)

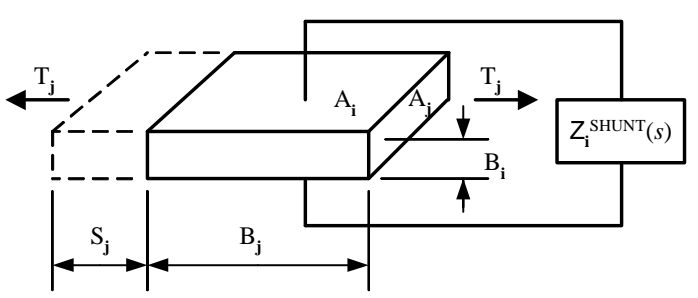

Figure 3. Model for a piezoelectric patch bounded to a shunt circuit.

where:

- PZT is the piezoelectric patch,

- $\mathrm{Z}_{\mathbf{i}}^{S H U N T}(s)$ is the electrical impedance of the shunt circuit $[\Omega]$,

- $\mathbf{i}$ and $\mathbf{j}$ give the coordinate axes that indicate the direction for electrical and mechanical vectors,

- $\mathrm{T}_{\mathbf{j}}$ is the stress $\left[\mathrm{N} / \mathrm{m}^{2}\right]$,

- $A_{i}$ and $A_{j}$ are the transversal section areas according to the vectors $\mathbf{i}$ and $\mathbf{j}\left[\mathrm{m}^{2}\right]$,

- $\mathrm{B}_{\mathbf{i}}$ and $\mathrm{B}_{\mathbf{j}}$ are the initial patch lengths according to vectors $\mathbf{i}$ and $\mathbf{j}[m]$,

- $\mathrm{S}_{\mathbf{j}}$ is the strain (non-dimensional), and

- $S$ is the Laplace variable.

As in Hagood and von Flotow (1991), the general expression that describes the behavior of piezoelectric materials, such as the one shown in Figure 3, is written as:

$$
\left[\begin{array}{l}
\mathbf{D} \\
\mathbf{S}
\end{array}\right]=\left[\begin{array}{ll}
\boldsymbol{\varepsilon}^{T} & \mathbf{d} \\
\mathbf{d}^{t} & \mathbf{s}^{E}
\end{array}\right]\left[\begin{array}{l}
\mathbf{E} \\
\mathbf{T}
\end{array}\right]
$$

where:

- $\mathbf{D}$ is the vector of electrical displacement $\left[\mathrm{C} / \mathrm{m}^{2}\right]$, 
- $\mathbf{E}$ is vector of electric field $[\mathrm{V} / \mathrm{m}]$,

- $\mathbf{S}$ is the vector of material engineering strains (non-dimensional),

- $\mathbf{T}$ is vector of material stresses $\left[\mathrm{N} / \mathrm{m}^{2}\right]$,

- $\varepsilon$ is the matrix of dielectric constants for the material $\left[C^{2} /\left(N \cdot m^{2}\right)\right]$,

- $\mathbf{s}$ is the matrix of compliance for the material $\left[\mathrm{m}^{2} / \mathrm{N}\right]$, and

- $\mathbf{d}$ is the piezoelectric material constant relating strain to voltage $[\mathrm{m} / \mathrm{V}]$.

Hagood and von Flotow (1991) also shows how to use Eq. (1) together with the basic equations of voltage and current from the electricity in order to obtain the following expression:

$$
\begin{aligned}
& {\left[\begin{array}{l}
\mathbf{I} \\
\mathbf{S}
\end{array}\right]=\left[\begin{array}{cc}
\mathbf{Y}^{E L E C T}(s) & s \mathbf{A d} \\
\mathbf{d}^{t} \mathbf{B}^{-1} & \mathbf{S}^{E}
\end{array}\right]\left[\begin{array}{l}
\mathbf{V} \\
\mathbf{T}
\end{array}\right] ;} \\
& \mathbf{Y}^{E L E C T}=\mathbf{Y}_{P Z T}^{D}+\mathbf{Y}^{S H U N T}
\end{aligned}
$$

where:

- $\mathbf{V}$ is the electric voltage,

- I is the electric current,

- B is the diagonal matrix of the lengths of the piezoelectric patch,

- A is the matrix of surfaces, perpendicular to the electric field,

- $\mathbf{Y}$ is the electric admittance, and

- $\mathbf{Y}_{P Z T}^{D}=s C_{P Z T}^{T}$.

At this time, it is important to define the piezoelectric constant known as electromechanical coupling coefficient, $k$. Hagood and von Flotow (1991) define this constant as being the relationship between the peak energy stored in the capacitor and the peak energy stored in the deformation of the material taking into account open electrodes for the piezoelectric patch. Physically, the square of this coefficient, $k^{2}$, represents the percentage of energy of mechanical deformation that can be turned into electric energy and vice-versa.

Mathematically, the electromechanical coupling coefficient is defined as:

$$
k_{\mathbf{i j}}=\frac{d_{\mathbf{i j}}}{\sqrt{s_{\mathbf{j} j}^{E} \varepsilon_{\mathbf{i}}^{T}}}
$$

where $d_{\mathbf{i j}}$ is the piezoelectric material constant relating voltage in the ith direction to strain in the $\mathbf{j t h}$ direction.

To close the modeling, Hagood and von Flotow (1991) show the mechanical impedance, in the non-dimensional form, for the piezoelectric patch with a shunt circuit as defined by the equation:

$$
\bar{Z}_{\mathbf{j} \mathbf{j}}^{M E C}(s)=\frac{Z_{\mathbf{j} \mathbf{j}}^{S H U N T}(s)}{Z_{\mathbf{j} \mathbf{j}}^{D}(s)}=\frac{1-k_{\mathbf{i j}}^{2}}{1-k_{\mathbf{i j}}^{2} \bar{Z}_{\mathbf{i}}^{E L E C T}(s)}
$$

where $Z_{\mathbf{j} \mathbf{j}}^{S H U N T}(s)$ is the mechanical impedance of the passive damping piezo device with the shunt circuit.

Finally, Hagood and von Flotow (1991) show that the frequency response function (FRF) that relates the acceleration and the external force of a resonant shunted piezoelectric in the Laplace domain is:

$$
\mathrm{H}(s)=\frac{s^{2}}{k_{r}+s Z_{\mathbf{j} \mathbf{j}}^{M E C}(s)+s^{2} m_{r}}
$$

This equation is valid for a single mode system, thus $m_{r}$ and $k_{r}$ are the modal mass and modal stiffness related to the mode $r$.

\section{Resonant Shunted Piezoelectrics - RSPs}

According to what has been seen up to now, the piezoelectric patches can be modeled as a capacitor in series with a controlled voltage source. Therefore, in order to obtain a resonant shunt circuit, the circuit shown in Figure 3 can be compound of an inductor and a resistor. The RL shunt circuit forms an RLC resonant network with the piezoelectric patch. This approach allows the dissipation of the energy associated with a given vibration mode. In this case, the strain energy (associated with the vibration) is converted into electric energy and dissipated in the form of heat, through the Joule effect. From the mechanical point of view, damping is introduced by appropriate tuning the resonance frequency of the RLC network to a given frequency related to one of the vibration modes of the mechanical structure.

\section{Series Resonant Shunt Circuit}

The resonant shunt circuit in series, as shown in Figure 4, was proposed by Hagood and von Flotow (1991). The same scheme can be found in Lesieutre (1998), Steffen and Inman (1999), Caruso (2001), Fleming et al. (2002), Fleming et al. (2003) and Park and Inman (2003).
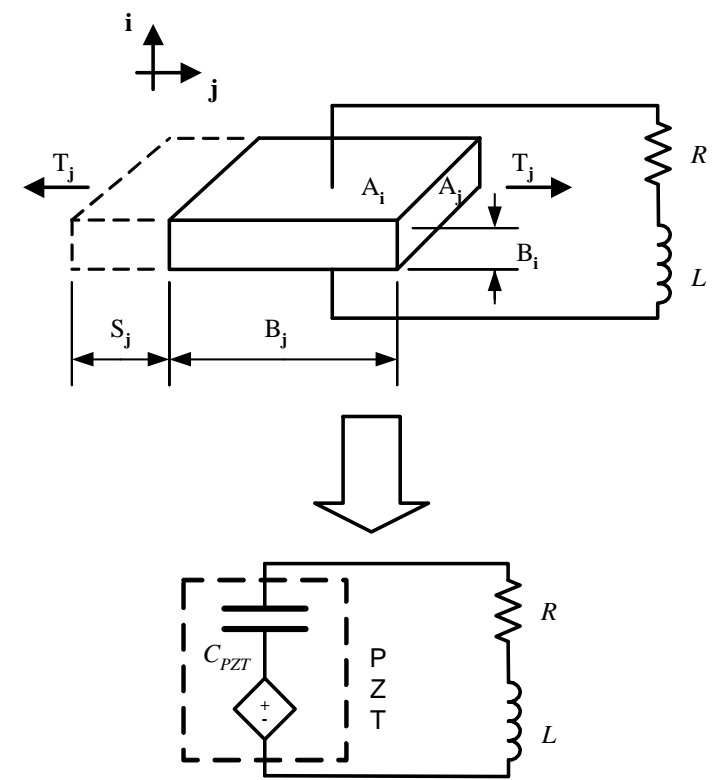

Figure 4. The series resonant shunt circuit.

An analysis of this circuit leads to the following relations:

$$
\begin{aligned}
& Y_{\mathbf{i}}^{S H U N T}(s)=\frac{1}{L s+R} ; \\
& \bar{Z}_{\mathbf{i}}^{E L E C T}(s)=\frac{L C_{P Z T}^{T} s^{2}+R C_{P Z T}^{T} s}{L C_{P Z T}^{T} s^{2}+R C_{P Z T}^{T} s+1}
\end{aligned}
$$

where:

- $L$ is the inductance,

- $R$ is the resistance, and 
- $C_{P Z T}^{T}$ is the inherent capacitance of the piezoelectric patch taken at constant stress (free).

Or, using Eq. (4), the non-dimensional impedance can be written as:

$$
\bar{Z}_{\mathbf{j} \mathbf{j}}^{R S P_{\text {SERIES }}}(s)=1-k_{\mathbf{i j}}^{2}\left(\frac{1}{L C_{P Z T}^{S} s^{2}+R C_{P Z T}^{S} S+1}\right)
$$

Equation (7) can be rewritten as:

$$
\bar{Z}_{\mathbf{j} \mathbf{j}}^{R S P_{\text {SERIES }}}(\gamma)=1-k_{\mathbf{i j}}^{2}\left(\frac{\delta^{2}}{\gamma^{2}+\delta^{2} \xi \gamma+\delta^{2}}\right)
$$

where:

- $\delta=\omega_{0} / \omega_{n}$ is the non-dimensional tuning ratio,

- $\omega_{0}=1 / \sqrt{L C_{P Z T}^{S}}$ is the electrical resonance frequency,

- $\omega_{n}=\sqrt{K / M}$ is the resonance frequency of the mechanical system,

- $\xi=R C_{P Z T}^{S} \omega_{n}^{E}$ is the damping factor,

- $\omega_{n}^{E}=\sqrt{\left(K+K_{\mathrm{jj}}^{E}\right) / M}$ is the resonance frequency of the mechanical system, corresponding to the open circuit case,

- $K$ is the stiffness of the mechanical system,

- $K_{\mathbf{j j}}^{E}=A_{\mathbf{j}} / s_{\mathbf{j j}}^{E} L_{\mathbf{j}}$ is the stiffness of the piezoelectric patch corresponding to the open circuit case, and

- $\gamma=s / \omega_{n}^{E}$ is the non-dimensional frequency.

Hagood and von Flotow (1991) point out that the parameters $\delta$ and $\xi$ are similar to those found for the DVA. Consequently, the series resonant shunt circuit can be designed by considering these similarities.

Figure 5 illustrates the two above-mentioned configurations for vibration reduction purposes. Figure 5-(a) shows a system containing a DVA; and Figure 5-(b) depicts a system containing an RSP.

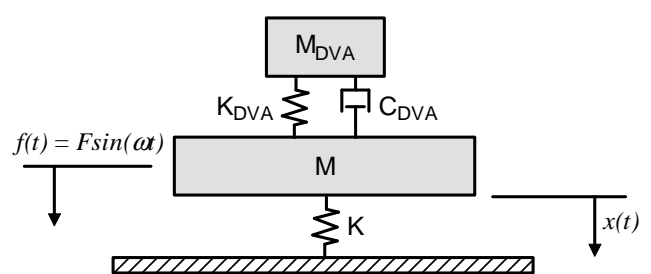

(a)

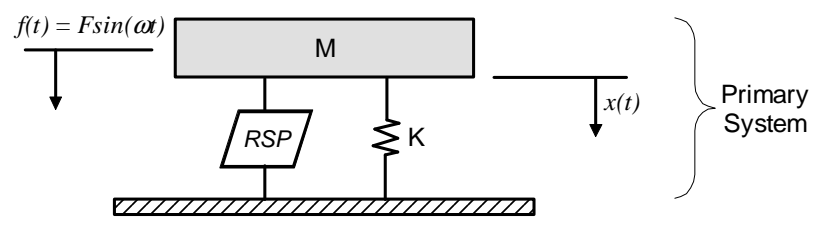

(b)

Figure 5. Comparison between systems with a DVA (a) and an RSP (b).
The generalized electromechanical coupling coefficient is defined as:

$$
K_{\mathrm{ij}}^{2}=\left(\frac{K_{\mathrm{jj}}^{E}}{K+K_{\mathrm{jj}}^{E}}\right)\left(\frac{k_{\mathrm{ij}}^{2}}{1-k_{\mathrm{ij}}^{2}}\right)=\bar{K} \frac{k_{\mathrm{ij}}^{2}}{1-k_{\mathrm{ij}}^{2}}
$$

where $\bar{K}$ is the ratio between the piezoelectric short circuit modal stiffness and the total system modal stiffness.

Now, it is possible to compare the frequency response functions for the DVA and the RSP, as calculated for the primary mass for both cases:

$$
\begin{aligned}
& H_{M_{P}}^{R S P_{\text {SERIES }}}(\gamma)=\frac{\left(\delta^{2}+\gamma^{2}\right)+\delta^{2} \xi \gamma}{\left(1+\gamma^{2}\right)\left(\delta^{2}+\gamma^{2}+\delta^{2} \xi \gamma\right)+K_{\mathrm{ij}}^{2}\left(\gamma^{2}+\delta^{2} \xi \gamma\right)} \\
& H_{M_{P}}^{D V A}(\gamma)=\frac{\left(\delta^{2}+\gamma^{2}\right)+\delta^{2} \xi \gamma}{\left(1+\gamma^{2}\right)\left(\delta^{2}+\gamma^{2}+\delta^{2} \xi \gamma\right)+\mu\left(\delta^{2} \gamma^{2}+\delta^{2} \xi \gamma^{3}\right)}
\end{aligned}
$$

Equations (10) and (11) highlight the similarities between the DVA and the RSP. It can be seen that the generalized electromechanical coupling coefficient, $K_{\mathbf{i j}}^{2}$, for the system with RSP series is similar to the relationship between the mass of the DVA and the mass of the primary structure, $\mu$, for the system with DVA. However, notice that the DVA operates by absorbing the kinetic energy associated to the vibration of the primary structure. The RSP, in a general way, dissipates a portion of the strain energy that is converted into electric energy. As a consequence, the optimal locations for each type of damping system are different: the DVA should be connected to a point of maximum displacement and the piezoelectric patch of an RSP should be bonded to a region of maximum deformation.

Similarly to the system with a DVA, the design of the optimal RSP series is obtained from the optimal tuning ratio, $\delta$, and the optimal damping factor, $\xi$, for the system. Therefore, these expressions are as below:

$$
\begin{aligned}
& \delta_{O P T M}^{R S P_{\text {SERIES }}}=\sqrt{1+K_{\mathrm{ij}}^{2}} \\
& \xi_{O P P_{S E R I E S}}^{R T M}=\frac{\sqrt{2} K_{\mathbf{i j}}}{\left(1+K_{\mathrm{ij}}^{2}\right)}
\end{aligned}
$$

Figure 6 illustrates the transfer function of the system with an RSP series, adopting $K_{\mathrm{ij}}^{2}=0.05, \delta=\delta_{O P T M}^{R S P_{S E R I E S}}$, and considering the non-dimensional frequency $g=\omega / \omega_{n}$, for different values of $\xi$. Similar to the system with a DVA, the two invariant points $\mathrm{P}$ and $\mathrm{Q}$ can be observed. Also, by increasing the value of the damping factor, the system behaves as a single degree of freedom one. 


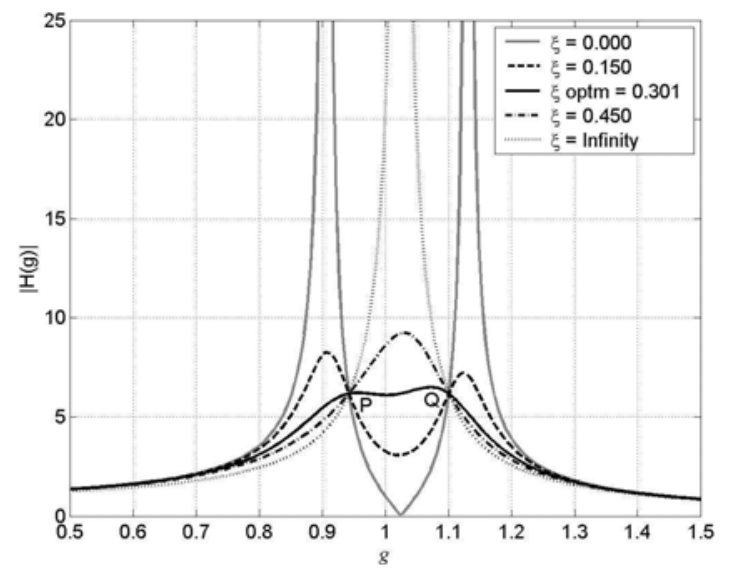

Figure 6. Transfer function of the primary mass for systems with an RSP series.

Finally, once the optimal parameters $\delta$ and $\xi$ for the RSP series are obtained, it is possible to determine the optimal values for the inductor and resistor of the shunt circuit, from the following equations:

$$
\begin{aligned}
& L_{O P T M}^{R S P_{S E R E S}}=\frac{1}{C_{P Z T}^{S} \omega_{0}^{2}}=\frac{1}{C_{P Z T}^{S}\left(\omega_{n} \delta_{O P T M}^{R S P_{S E R E S}}\right)^{2}}=\frac{1}{C_{P Z T}^{S}\left(1+K_{\mathrm{ij}}^{2}\right) \omega_{n}^{2}} \\
& R_{O P T M}^{R S P_{\text {SERES }}}=\frac{\xi_{O P S M}^{R S P_{\text {SERES }}}}{C_{P Z T}^{S} \omega_{n}}=\frac{\sqrt{2} K_{i j}}{C_{P Z T}^{S} \omega_{n}\left(1+K_{\mathrm{ij}}^{2}\right)}
\end{aligned}
$$

\section{Parallel Resonant Shunt Circuit}

The parallel resonant shunt circuit (see Figure 7) was first proposed by $\mathrm{Wu}$ (1996), aiming at overcoming implementation difficulties of the series circuit. The practical aspects regarding the two circuit topologies will be discussed in the later sections.
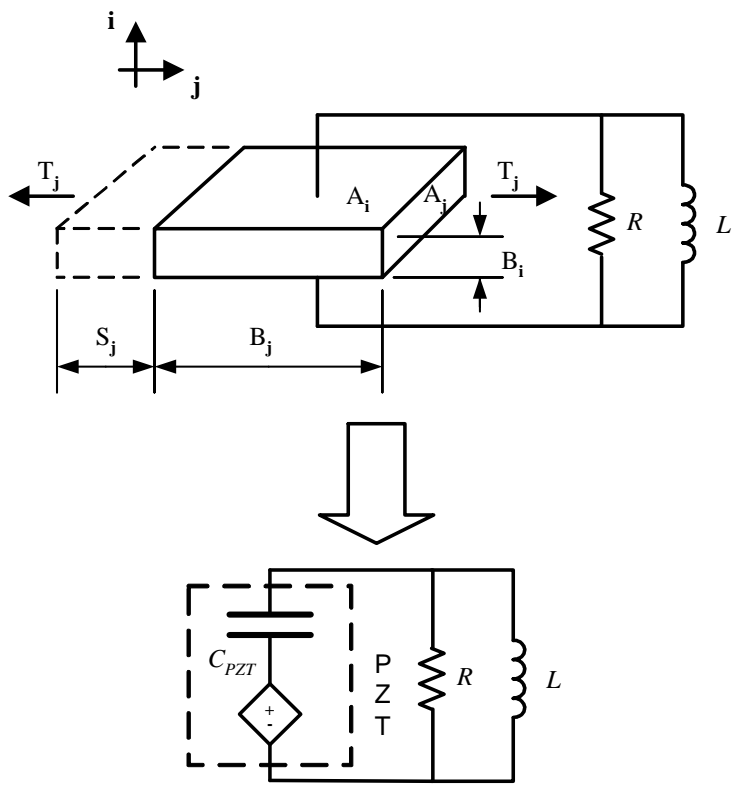

Figure 7. The parallel resonant shunt circuit.
As in the series circuit, the analysis of the electrical circuit leads to the following equations:

$$
\begin{aligned}
& Y_{\mathbf{i}}^{S H U N T}(s)=\frac{1}{L s}+\frac{1}{R} \\
& \bar{Z}_{\mathbf{i}}^{E L E C T}(s)=\frac{L R C_{P Z T}^{T} s^{2}}{R L C_{P Z T}^{T} s^{2}+L s+R}
\end{aligned}
$$

And, consequently:

$$
\begin{aligned}
& \bar{Z}_{\mathbf{j} \mathbf{j}}^{R S P_{P A R A L L E L}}(s)=1-k_{\mathbf{i j}}^{2}\left(\frac{L s^{2}+R}{R L C_{P Z T}^{S} s^{2}+L s+R}\right) \\
& \bar{Z}_{\mathbf{j} \mathbf{j}}^{R S P_{P A R A L L E L}}(s)=1-k_{\mathbf{i j}}^{2}\left(\frac{\gamma+\xi \delta^{2}}{\xi \gamma^{2}+\gamma+\xi \delta^{2}}\right)
\end{aligned}
$$

Using Eq. (15) and the proper modeling of the single DOF system, it is possible to obtain the transfer function as:

$$
H_{M_{P}}^{R S P_{P A R A L L L L}}(\gamma)=\frac{\left(\delta^{2} \xi+\gamma^{2} \xi+\gamma\right)}{\left(\delta^{2} \xi+\gamma^{2} \xi+\gamma\right)\left(1+\gamma^{2}\right)+K_{\mathbf{i j}}^{2} \gamma^{2} \xi}
$$

$\mathrm{Wu}$ (1996) presents details for obtaining the optimal parameters of the circuit. Similarly to Hagood and von Flotow (1991), he also makes use of the transfer function to draw his conclusions. This way, the expressions are:

$$
\begin{aligned}
& \delta_{O P T M}^{R S P_{P A R A L L L L}}=\sqrt{1+\frac{K_{\mathbf{i j}}^{2}}{2}} \\
& \xi_{O P T M}^{R S P_{P A R A L E L}}=\frac{1}{\sqrt{2} K_{\mathrm{ij}}}
\end{aligned}
$$

And consequently:

$$
\begin{aligned}
& L_{O P T M}^{R S P_{P A R A L L L L}}=\frac{1}{C_{P Z T}^{S}\left(1-\frac{K_{\mathrm{ij}}^{2}}{2}\right) \omega_{n}{ }^{2}} \\
& R_{O P T M}^{R S P_{P A R A L E L}}=\frac{1}{\sqrt{2} K_{\mathrm{ij}} C_{P Z T}^{S} \omega_{n}}
\end{aligned}
$$

Figure 8 shows the transfer functions of the system with an RSP

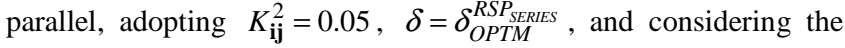
non-dimensional frequency $g=\omega / \omega_{n}$, for different values of $\xi$. 


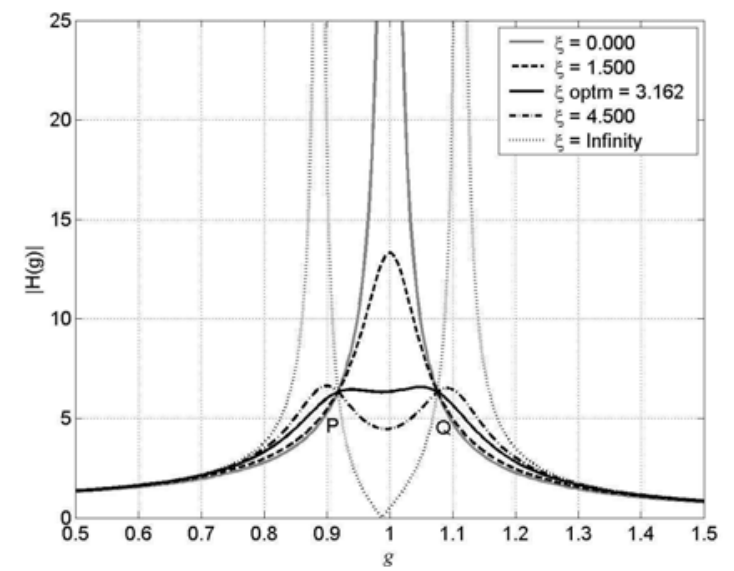

Figure 8. Transfer function of the primary mass for systems with an RSP parallel.

\section{Electric Analysis of the Shunted Piezoelectric Components}

The following sections show the analysis of the piezoelectric patch and the synthetic inductors from the electrical viewpoint. For the piezoelectric patch, the associated impedance and its behavior are verified when bonded to a vibratory structure. Two basic types of synthetic inductors are presented.

\section{The Piezoelectric Patch}

As an example, the manufacturer (Mide Technology Corporation) provides the information presented in Tab. 3 about the ACX QP15N piezoelectric patch.

Table 3. ACX QP15N specifications.

\begin{tabular}{|c|c|c|}
\hline Size $[\mathrm{mm}]$ & Weight[g] & $\begin{array}{c}\text { Number of } \\
\text { elements }\end{array}$ \\
\hline $50.8 \times 25.4 \times 0.254$ & 2.268 & 1 piezoelectric patch \\
\hline $\begin{array}{c}\text { Piezoelectric patch } \\
\text { size [mm] }\end{array}$ & Capacitance [nF] & $\begin{array}{c}\text { Operation range } \\
{[\text { V] }}\end{array}$ \\
\hline $\begin{array}{c}45.974 \times 20.574 \times \\
0.127\end{array}$ & 100 & \pm 100 \\
\hline
\end{tabular}

However, for the system compound by an ACX QP15N bonded to a flexible beam as shown in Figure 9 , by using an impedance analyzer (Hewlett Packard HP4194A), a variation of the impedance with respect to the frequency is found, as shown by Figure 10 .

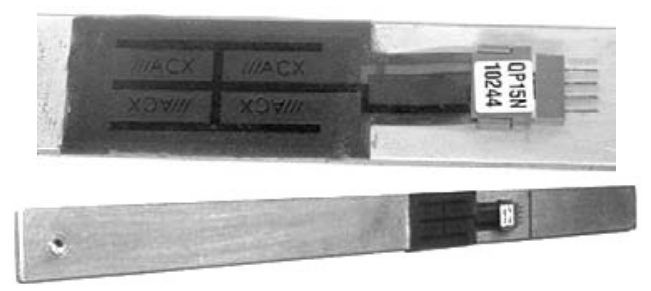

Figure 9. ACX QP15N + Beam System.

Figure 10 shows also that impedance is not purely reactive. The real part of the impedance, Figure 10-(a), introduces a resistive element. Besides, the imaginary part of the impedance, Figure 10-(b), confirms the capacitive element. From the phase curve, Figure 10(d), the predominance of the capacitive component is verified, since the values obtained are close to $-90^{\circ}$. The inherent impedance of the piezoelectric patch can be viewed as a resistor, Figure 10-(e), which is in series with a capacitor, Figure 10-(f).
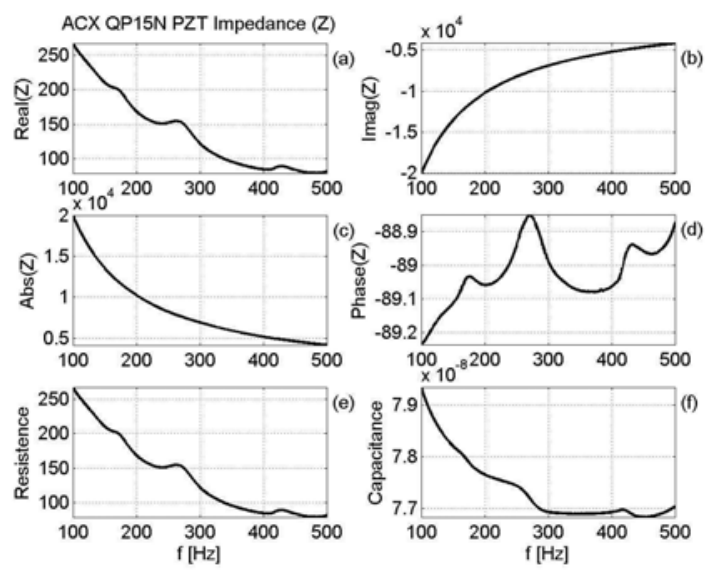

Figure 10. Impedance for the ACX QP15N + Beam system.

To validate the above-presented model, the ACX QP15N + beam system is tested using the setup shown in Figure 11.

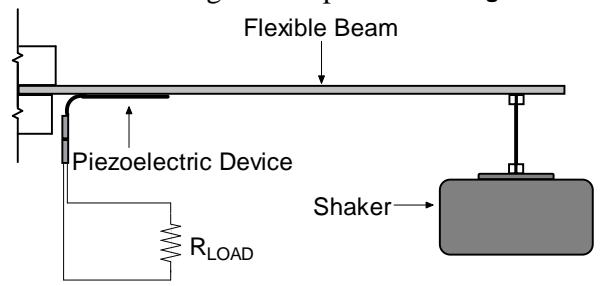

Figure 11. Experimental setup for the verification of the electrical characteristics of the ACX QP15N + Beam + RLOAD system.

A shaker excites the system according to a predefined frequency and amplitude. Therefore, according to the modeling of the piezoelectric device, the controlled voltage source (internal to the patch) also produces voltage. Electrically, the set constituted by the piezoelectric patch and load resistance forms a circuit characterized by a voltage source, a capacitor, a resistor and a load resistance, as shown in Figure 12.

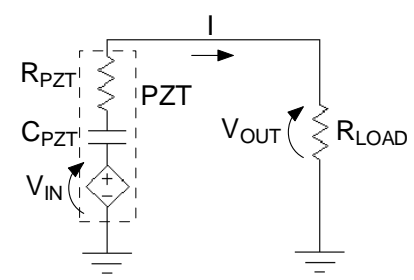

Figure 12. Resultant electric circuit from the ACX QP15N + Beam + RLOAD system.

This way, the current and the electric power on $R_{L O A D}$ can be calculated by using the Ohm's law:

$$
\begin{gathered}
I(\omega)=\frac{V_{I N}(\omega)}{Z_{T O T A L}(\omega)}=\frac{V_{O U T}(\omega)}{R_{L O A D}} \\
P_{L O A D}=V_{O U T}^{R M S} I^{R M S}=\frac{\left(V_{\text {OUT }}^{R M S}\right)^{2}}{R_{\text {LOAD }}}
\end{gathered}
$$


Experimentally, the voltage $V_{I N}(\omega)$ can be obtained through an oscilloscope, by keeping the terminals of the piezoelectric element in open circuit, as illustrated in Figure 13. $V_{I N}(\omega)$ depends on the magnitude of the excitation provided by the shaker. As the excitation remains the same, $V_{I N}(\omega)$ is not altered.

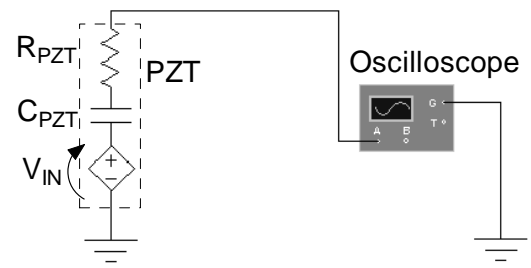

Figure 13. Setup for the experimental obtaining of $V_{\mathbb{I N}}(\omega)$.

Consequently, by varying the load resistance and measuring the voltage in the load resistance, the curves corresponding to $V_{O U T} \times R_{L O A D}, I \times R_{L O A D}$ and $P_{L O A D} \times R_{L O A D}$ can be generated. Figure 14 illustrates the comparison of the experimental data with those obtained from the analytical model of the proposed electric circuit.
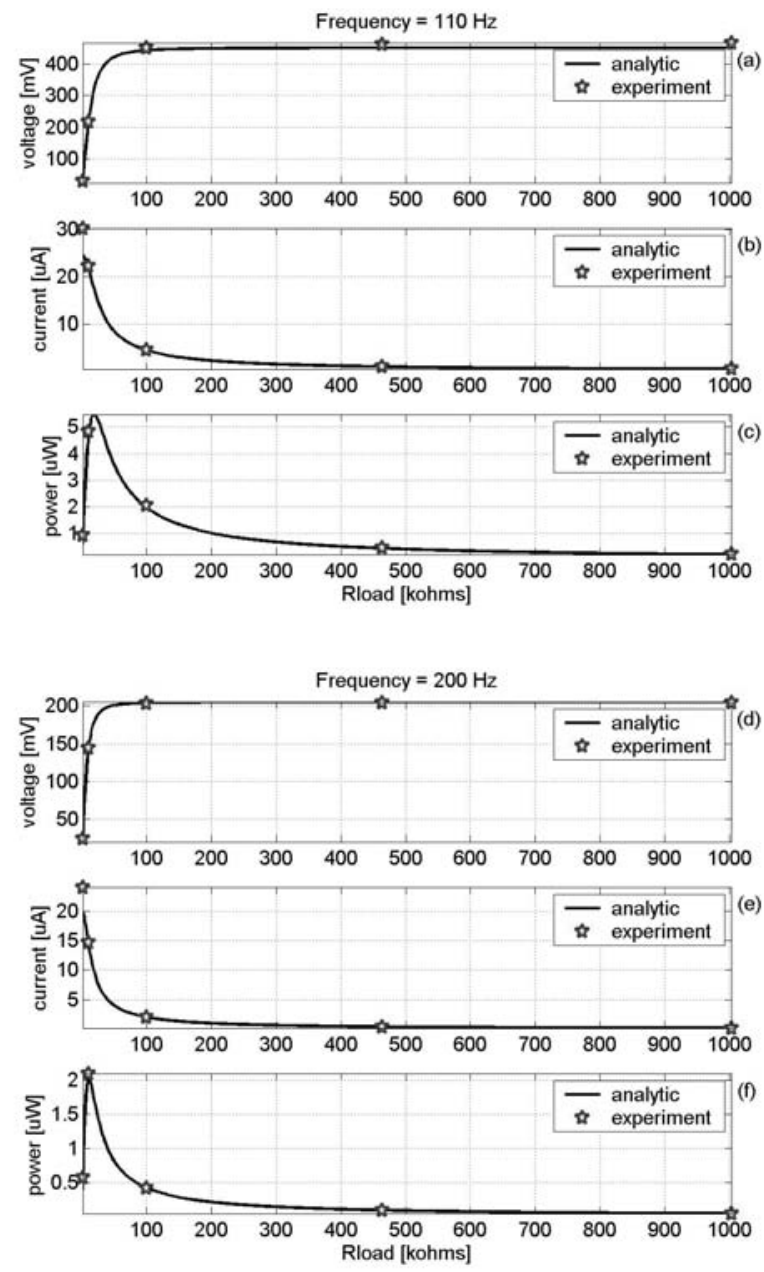

Figure 14. Experimental versus analytical data for the ACX QP15N + Beam $+\mathbf{R}_{\text {LOAD }}$ system.

\section{The Resonant Shunt Circuit and the Synthetic Inductor}

Equations (13) and (18) show that the values of the inductance are inversely proportional with respect to the angular frequency. In the real cases, the associated frequencies are relatively low. As a consequence, the resonant shunt circuits require large values for the inductance. These values would typically attain hundreds of henries. The weight and the volume of such inductors would make unfeasible the use of this technique. To overcome this limitation, synthetic inductors are obtained by using operational amplifiers, as in previous works (Riordan, 1967; Antoniou, 1969; Stephenson, 1985; Schaumann et al., 1990; Massara et al., 2000 and Park and Inman, 2003). Even assuming different configurations, these circuits are known as synthetic inductors or gyrators. In the present contribution, two types of synthetic inductors were explored, namely the one proposed by Antoniou (1969), and the other one based on Riordan (1967). For the sake of simplicity, they are called Antoniou synthetic inductor and Riordan synthetic inductor, respectively.

\section{Antoniou Synthetic Inductor}

Figure 15 shows the synthetic inductor circuit as proposed by Antoniou (1969).

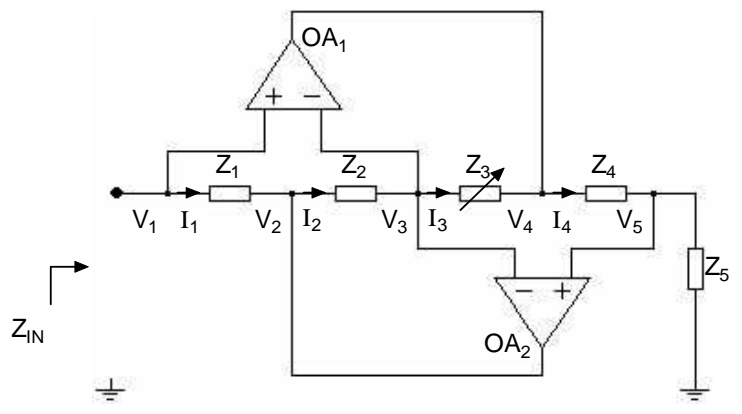

Figure 15. Circuit for the Antoniou synthetic inductor.

The input impedance is given by equation (20):

$$
Z_{I N}=\frac{Z_{1} Z_{3} Z_{5}}{Z_{2} Z_{4}}
$$

From the previous equation, a synthetic inductor is obtained by using the following relations: $Z_{4}=-j / \omega C_{4}, Z_{1}=R_{1}, Z_{2}=R_{2}$, $Z_{3}=R_{3}$ and $Z_{5}=R_{5}$. The equivalent circuit impedance $Z_{I N}$ is the same as an inductor $\left(L_{e q}\right)$, as shown below:

$$
\begin{aligned}
& Z_{I N}=j \omega L_{e q} ; \\
& L_{e q}=\frac{R_{1} R_{3} C_{4} R_{5}}{R_{2}}
\end{aligned}
$$

\section{Riordan Synthetic Inductor}

Figure 16 shows the synthetic inductor circuit as proposed by Riordan (1967). 


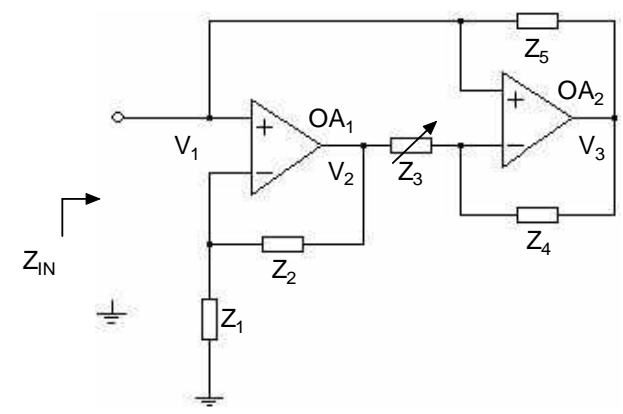

Figure 16. Circuit for the Riordan synthetic inductor.

In despite of presenting a different topology, the equations for the input impedance are the same as those of the Antoniou synthetic inductor.

\section{Practical Aspects about the Synthetic Inductors}

In order to test the performance of synthetic inductors at low electrical frequencies (as typically found in most mechanical systems), experiments simulating the resonant shunt circuit were performed. An RLC series filter was configured in this experiment by using the synthetic inductor together with a resistor $R_{0}$, a capacitor $C_{P Z T}$ and a signal generator (Brüel \& Kjær Sine/Noise Generator Type 1049). The filter is described by Eq. (22), which relates the voltage in $R_{0}$ and the voltage in the generator. A signal analyzer (Spectral Dynamics SD380) was used to perform the frequency analysis of the circuit. The setup of the experiment is shown in Figure 17.

$$
H_{L O A D}(\omega)=\frac{1}{1+j \frac{1}{R}\left(\omega L-\frac{1}{\omega C_{P Z T}}\right)}
$$

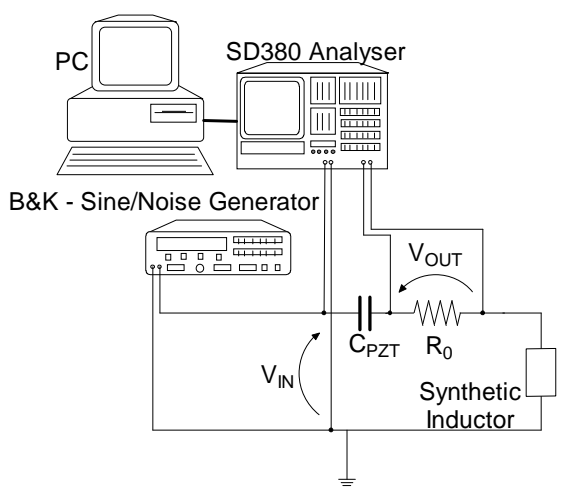

Figure 17. Experimental setup to test the synthetic inductors.

The signal generator introduces in the circuit a white noise of bandwidth $2 \mathrm{~Hz}$, to $2 \mathrm{kHz}$, with $1.25 \mathrm{~V}$ of RMS value. The signals were acquired simultaneously in a sample of $T=1.6 \mathrm{~s}$, with intervals of $d t=0.78125 \mathrm{~ms}$. Then the maximum frequency analyzed is fmax $=500 \mathrm{~Hz}$ and the frequency resolution is $d f=0.625 \mathrm{~Hz}$. The transfer function was estimated by using 50 samples.

The capacitors $C_{4}$ and $C_{P Z T}$ and the resistor $R_{0}$ were fixed to $108.5 n F, 110.1 n F$ and $9.98 k \Omega$, respectively. Table 4 shows the values used for the remaining components along the experiments. In this case, the inductance is calculated according to the equation:

$$
L_{e q}=\frac{1}{\left(2 \pi f_{\text {elet }}\right)^{2} C_{P Z T}}
$$

Table 4. Experimental values for $\mathbf{R}_{1}, \mathbf{R}_{2}, \mathbf{R}_{3}$ and $\mathbf{R}_{5}$ and the calculated one for $L_{\text {eq. }}$.

\begin{tabular}{|c|c|c|c|c|c|}
\hline Experiment & $R_{1}[k \Omega]$ & $R_{2}[k \Omega]$ & $R_{3}[k \Omega]$ & $R_{5}[k \Omega]$ & $L_{e q}[H]$ \\
\hline$\# \mathbf{1}$ & 2.18 & 2.17 & 97.7 & 2.16 & 23.00 \\
\hline$\# \mathbf{2}$ & 5.51 & 5.52 & 38.6 & 5.51 & 23.03 \\
\hline$\# \mathbf{\# 4}$ & 14.78 & 14.68 & 14.35 & 14.67 & 22.0 \\
\hline$\# \mathbf{5}$ & 46.2 & 46.2 & 4.61 & 46.0 & 23.01 \\
\hline$\# \mathbf{6}$ & 219 & 220 & 0.964 & 221 & 23.01 \\
\hline $\mathbf{7 6}$ & 330 & 329 & 0.643 & 329 & 23.02 \\
\hline
\end{tabular}

Figure 18 and Figure 19 present the graphics of the amplitude, phase and coherence of the transfer function for the two types of synthetic inductors. A comparison between the two types of synthetic inductors does not allow any straightforward conclusion about the superiority of one with respect to the other. However, by taking into account the graphics of the module and phase, it is possible to notice good repeatability for the tests, since the resonance frequency remains approximately the same for all the experiments. The graphics of the coherence indicate that along the experiments the values of the coherence become worse, probably due to the values of the resistors. Finally, it is also possible to notice that the electric network available contaminates the experiments in $60 \mathrm{~Hz}$ (used in Brazil) and in some other harmonics in all the experiments.
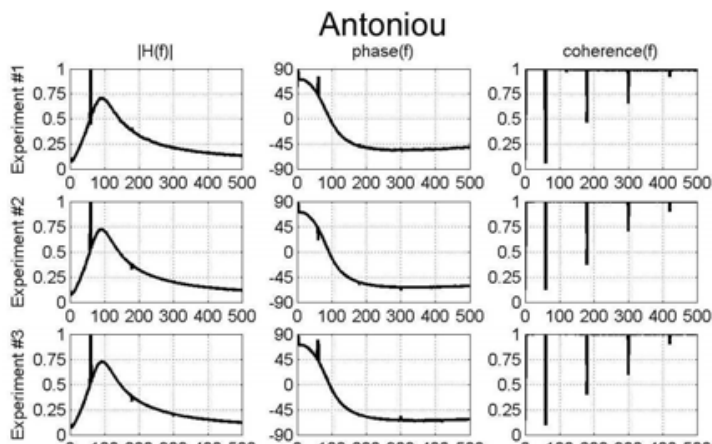

100200300400500
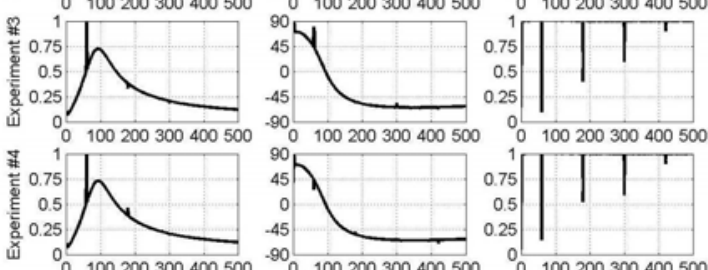

急
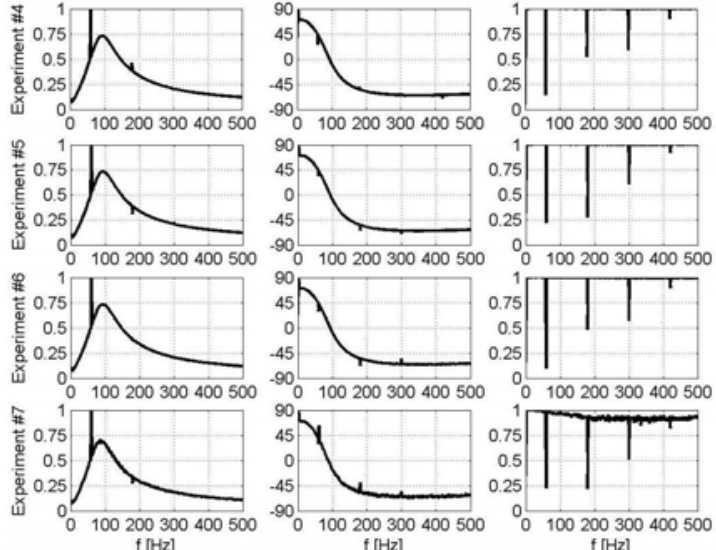

Figure 18. Amplitude, phase and coherence of the transfer function for Antoniou synthetic inductor. 

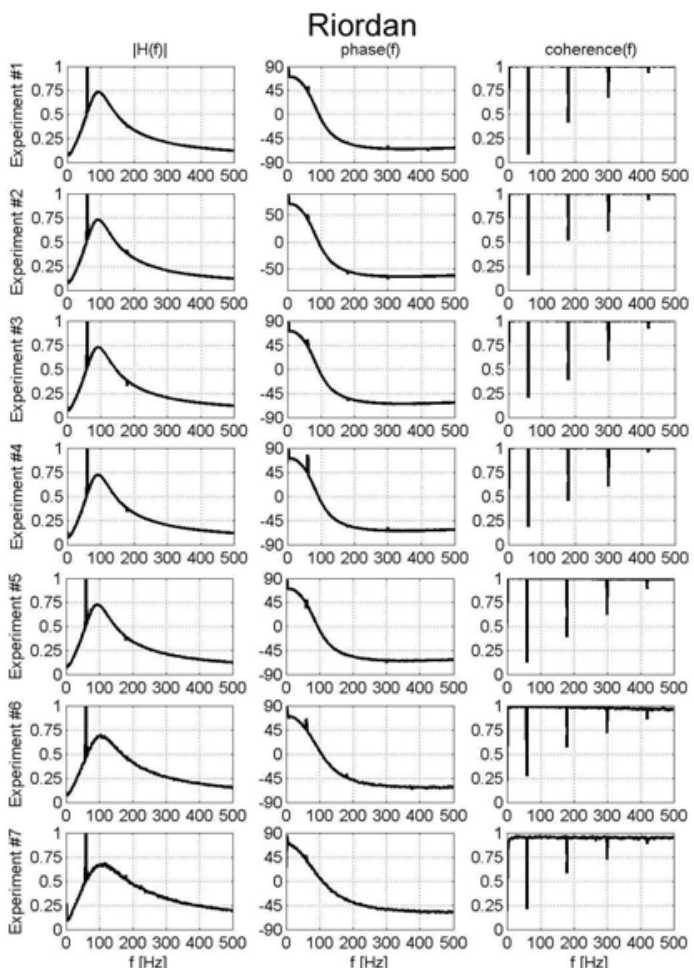

$\mathrm{t}[\mathrm{Hzl}$

Figure 19. Amplitude, phase and coherence of the transfer function for Riordan synthetic inductor.

Figure 18 and Figure 19 also show a discrepancy between the experimental results and the analytical model adopted for the RLC circuit, as described by Eq.(22). For example, in all cases the gains are not unitary in the resonant frequency. It can be concluded that the non-idealities of the synthetic inductor generate one or more parasite resistances associated with the equivalent inductor. Park et al (2003) discussed about an experiment that evaluates the impedance of the synthetic inductor. By using an impedance analyzer, it was shown the existence of a frequency dependent inherent resistance in the synthetic inductor.

In this work, it is desired to obtain an equivalent model for the synthetic inductor. To achieve this aim, four different configurations were tested as candidates for the equivalent circuit used in the experiments, as shown in Figure 20.

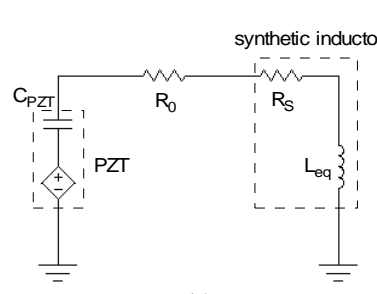

(a)

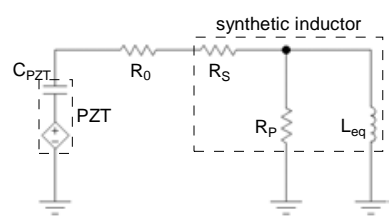

(c)
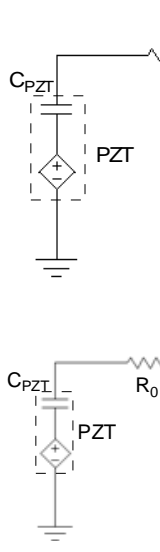

(b)

synthetic inductor

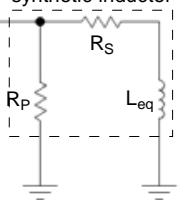

Figure 20. Candidate configurations to the synthetic inductor equivalent circuit (a) a resistor $R_{S}$ in series with an inductor $L_{e q}$, (b) a resistor $R_{p}$ in parallel with an inductor $L_{e q}$,(c) a resistor $R_{p}$ in parallel with an inductor $L_{e q}$ and a resistor $R_{S}$ in series with this branch and (d) a resistor $R_{S}$ in series with an inductor $L_{e q}$ and a resistor $R_{P}$ in parallel with this branch.
Experiment \#4 for the Riordan synthetic inductor was arbitrarily chosen to check which model better describes the behavior of the real circuit. From a curve fitting procedure, it was possible to obtain the values of $R_{S}$ and $R_{P}$, as presented in Table 5 and Figure 21 .

Table 5. Values of $\mathbf{R}_{\mathrm{S}}$ and $\mathbf{R}_{\mathrm{p}}$.

\begin{tabular}{|c|c|c|c|c|c|}
\hline $\begin{array}{c}\text { Circuit } \\
\text { Figure } \\
20-(\mathrm{a})\end{array}$ & $\begin{array}{c}\text { Circuit } \\
\text { Figure } \\
20-(\mathrm{b})\end{array}$ & \multicolumn{2}{|c|}{$\begin{array}{c}\text { Circuit } \\
\text { Figure 20-(c) }\end{array}$} & \multicolumn{2}{c|}{$\begin{array}{c}\text { Circuit } \\
\text { Figure 20-(d) }\end{array}$} \\
\hline$R_{S}$ & $R_{P}$ & $R_{S}$ & $R_{P}$ & $R_{S}$ & $R_{P}$ \\
{$[k \Omega]$} & {$[k \Omega]$} & {$[k \Omega]$} & {$[k \Omega]$} & {$[k \Omega]$} & {$[k \Omega]$} \\
\hline 4.969 & 72.495 & 3.067 & 140.591 & 3.506 & $5.398 \times 10^{11}$ \\
\hline
\end{tabular}
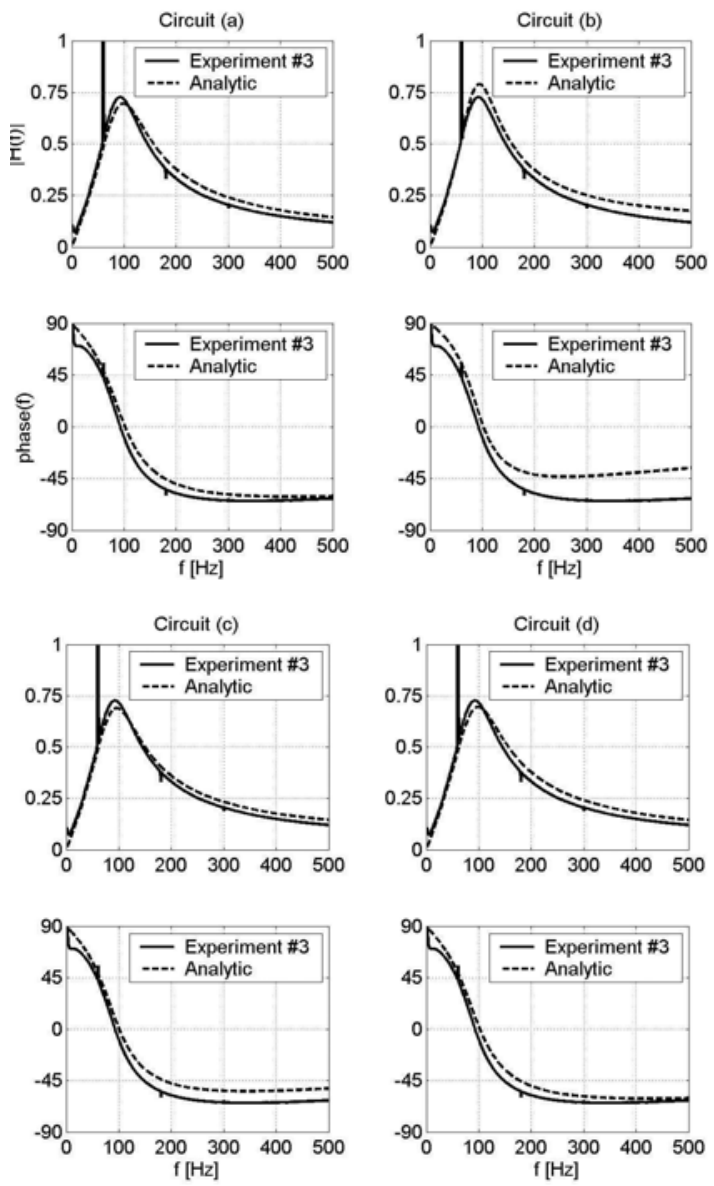

Figure 21. Amplitude and phase of the experimental and analytical transfer functions.

By analyzing Table 5 and Figure 21, it can be concluded that the best representation of the real electrical circuit is given by the circuit (a), for which the synthetic inductor is characterized as a resistor $R_{S}$ in series with an equivalent inductor $L$, as described by Eq. (22). Circuit (d) presents very similar results, however, the value of the resistor $R_{P}$ is extremely high, suggesting that $R_{P}$ tends to infinite (open circuit). This characteristic automatically leads to the choice of circuit (a). In this work, the inherent resistance of the synthetic inductor is called $R_{\text {PARASITE }}$.

Once defined the model for the synthetic inductor, a second set of experiments verifies the behavior of the inherent resistance as a function of the value of the equivalent inductor, both for the Antoniou and Riordan synthetic inductors. In the experiments, the 
values of $R_{0}$ and $C_{0}$ were fixed to $9.86 \mathrm{k} \Omega$ and $111.5 \mathrm{nF}$, respectively, and a number of different values for the resonance frequency of the circuit, $f_{0}$, was predefined. Consequently, the value of the inductor varies, leading to the variation of $R_{\text {PARASITE }}$. For both considered inductors the values of $R_{0}, R_{1}, R_{2}, R_{5}, C_{0}$ and $C_{4}$ were fixed to $9.86 \mathrm{k} \Omega, 46.2 \mathrm{k} \Omega, 46.2 \mathrm{k} \Omega, 46.0 \mathrm{k} \Omega, 111.5 \mathrm{nF}$, and $112.6 n F$, respectively.

The results can be seen in Table 6 and Figure 22 .

Table 6. Experimental value for $f_{0}, L_{e q}$ and $R_{\text {PARASITE. }}$

\begin{tabular}{|c|c|c|c|}
\hline \multicolumn{5}{|c|}{ Antoniou Synthetic Inductor } \\
\hline Experiment & $f_{0}[\mathrm{~Hz}]$ & $L[\mathrm{H}]$ & $R_{\text {PARASITE }}[\mathrm{k} \Omega]$ \\
\hline$\# 1$ & 396.25 & 1.3573 & 0.9553 \\
\hline$\# 2$ & 301.25 & 2.3175 & 1.1314 \\
\hline$\# 3$ & 198.75 & 5.3116 & 1.7849 \\
\hline$\# 4$ & 100.00 & 20.6205 & 4.0478 \\
\hline$\# 5$ & 49.375 & 81.4147 & 10.9426 \\
\hline$\# 6$ & 22.50 & 368.4433 & 39.4855 \\
\hline \multicolumn{5}{|c|}{ Riordan Synthetic Inductor } \\
\hline Experiment & $f_{0}[\mathrm{~Hz}]$ & $L[\mathrm{H}]$ & $R_{\text {PARASITE }}[\mathrm{k} \Omega]$ \\
\hline$\# 1$ & 401.875 & 1.4682 & 1.0776 \\
\hline$\# 2$ & 297.500 & 2.5302 & 1.2170 \\
\hline$\# 3$ & 200.000 & 5.3308 & 1.7967 \\
\hline$\# 4$ & 99.375 & 20.5834 & 4.2154 \\
\hline$\# 5$ & 48.750 & 81.5618 & 11.1978 \\
\hline$\# 6$ & 23.125 & 368.1111 & 37.6879 \\
\hline
\end{tabular}
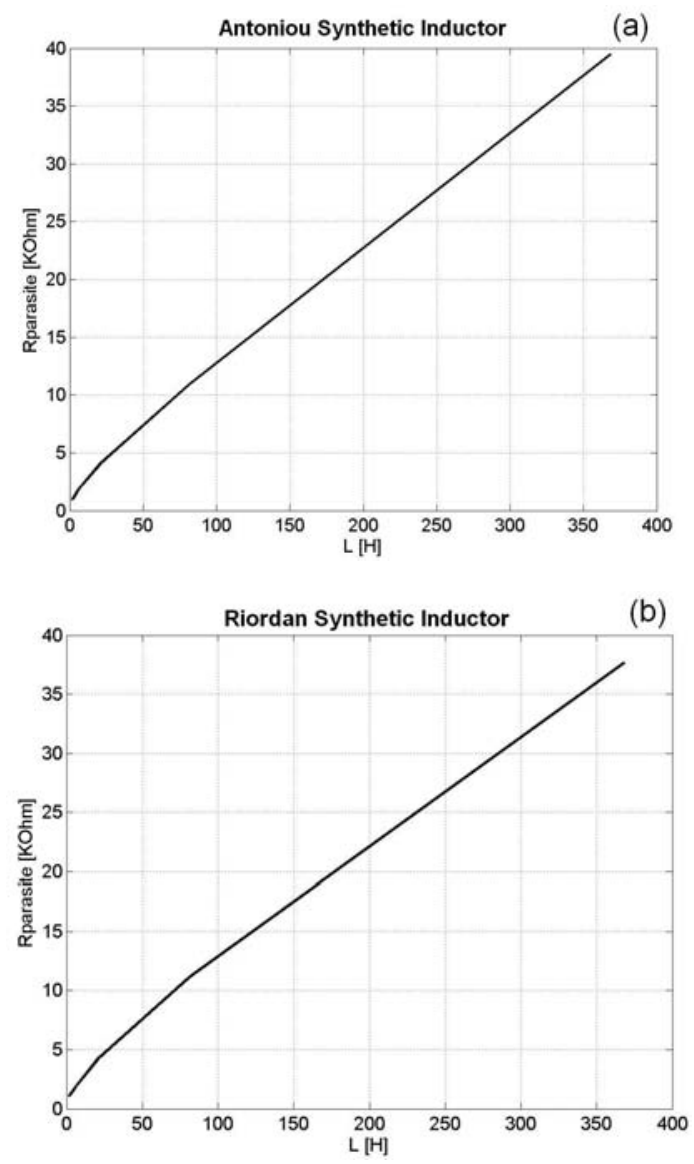

Figure 22. $R_{\text {PARASTE }}$ versus $L$ for the synthetic inductors.
Both for the Antoniou and for the Riordan synthetic inductors, it is easy to notice that the value of the parasite resistance increases when the inductance values also increase. This behavior is quite linear along the analyzed range. This means that, by taking into account a pre-defined value for the capacitance, the RLC filters exhibit better performance when designed to operate at larger electrical resonance frequencies. This fact has a direct consequence in shunted piezoelectric applications, since the frequencies of interest in these cases are rather low from the electrical point of view.

\section{Multiple Degree-of-Freedom (MDOF) Systems}

Real systems are continuous and non-homogeneous, which means that an infinite number of degrees of freedom should be considered to represent them, accordingly. Therefore, the analysis of this type of systems is only possible through analytic (exact) models. Unfortunately, the solution of these models is not easily obtained. Thus, it is necessary to use approximate techniques that describe the behavior of the system by using a finite number of degrees of freedom (Maia and Silva, 1997). To exemplify, consider the undamped N DOF system shown in Figure 23.

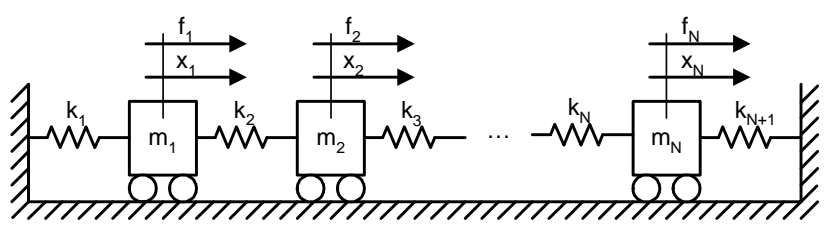

Figure 23. Discrete model for an $\mathrm{N}$ degrees of freedom system.

The equation of motion can be written in the matrix notation as:

$$
\left[\mathbf{K}-\omega^{2} \mathbf{M}\right] \mathbf{x}=\mathbf{f}
$$

By taking $\mathbf{f}=0$, it is possible to obtain the modal model for the free system, which is commonly expressed by the following pair of $\mathrm{NxN}$ matrices:

$$
\begin{aligned}
& {\left[\omega_{r}^{2}\right]=\left[\begin{array}{cccc}
\omega_{1}^{2} & 0 & \cdots & 0 \\
0 & \omega_{2}^{2} & \cdots & 0 \\
\vdots & \vdots & \ddots & \vdots \\
0 & 0 & \cdots & \omega_{N}^{2}
\end{array}\right]} \\
& \boldsymbol{\psi}=\left[\begin{array}{llll}
\left\{\psi_{1}\right\} & \left\{\psi_{2}\right\} & \cdots & \left\{\psi_{N}\right\}
\end{array}\right]
\end{aligned}
$$

The eigenvalues $\omega_{1}^{2}, \omega_{2}^{2}, \ldots, \omega_{N}^{2}$ represent the natural frequencies of the undamped system. The eigenvector matrix $\psi$ (also known as the modal matrix of the system), is compound by the modal shapes given by $\left\{\psi_{r}\right\}(\mathrm{r}=1,2, \ldots, \mathrm{N})$.

In order to find the FRF of the system, it is necessary to perform the following matrix transformations:

$$
\begin{aligned}
& {[\boldsymbol{\psi}]^{t}[\mathbf{M}][\boldsymbol{\psi}]=\left[{ }^{-} m_{r}{ }^{\cdot}\right]} \\
& {[\psi]^{t}[\mathbf{K}][\Psi]=\left[k_{r}{ }^{\prime}\right]}
\end{aligned}
$$

where $m_{r}$ and $k_{r}$ are known as modal mass and modal stiffness, respectively. 
The FRF that relates the acceleration obtained at the position $i$ when an external force is applied at the position $k$ is given by the following equation:

$$
\mathrm{H}_{\mathrm{ik}}(s)=\sum_{r=1}^{N} s^{2} \frac{\psi_{\mathrm{i} r} \psi_{\mathrm{k} r}}{k_{r}+s^{2} m_{r}}
$$

For more details about MDOF systems refer to Maia and Silva (1997).

\section{Modeling of Shunted Piezoelectrics for MDOF Systems}

As previously shown in Viana (2005), it is possible to note the similarity between Eq. (5), which describes the system of 1 DOF containing the shunted piezoelectric device and Eq.(27), which describes a MDOF system. It can be observed that Eq. (27) takes into account the influence of the $\mathrm{N}$ modes in the system response.

This way, by considering the analyses above, the effect of the piezoelectric patches and their shunt circuits can be easily introduced in the MDOF system. This can be achieved by adding the term $Z_{\mathbf{j} \mathbf{j}}^{M E C_{r}}(s)$ in the denominator of Eq. (27). Mathematically:

$$
\mathrm{H}_{\mathrm{ik}}(s)=\sum_{r=1}^{N} s^{2} \frac{\psi_{\mathrm{i} r} \psi_{\mathrm{k} r}}{k_{r}+s Z_{\mathbf{j} \mathbf{j}}^{M E C_{r}}(\omega)+s^{2} m_{r}}
$$

It is worth mentioning that $m_{r}$ and $k_{r}$ in Eq. (28) include the influence of the PZT (Pereira, 2003).

As an example, consider the case as depicted in Figure 24. This figure illustrates an undamped flexible beam (a) containing two piezoelectric passive damping devices (b). A PZT and its corresponding shunt circuit characterize each passive device. Damping in this case is designed to reduce the vibration amplitudes of two pre-defined modes, simultaneously.
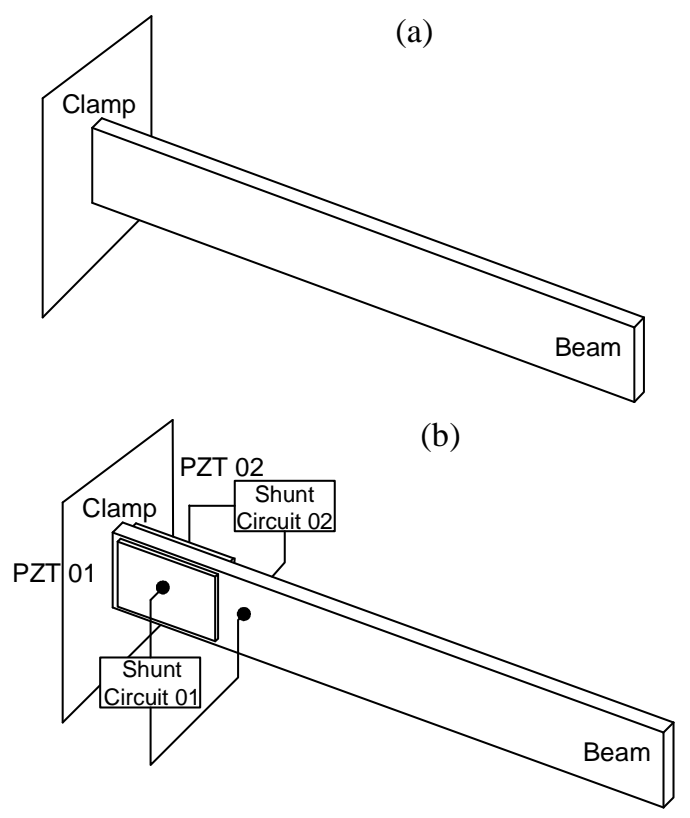

Figure 24. Beam (a) without and (b) with piezoelectric shunt devices.

Figure 25 shows the FRF obtained numerically. The circuits were designed to reduce the vibration amplitudes of the second and third modes, simultaneously.

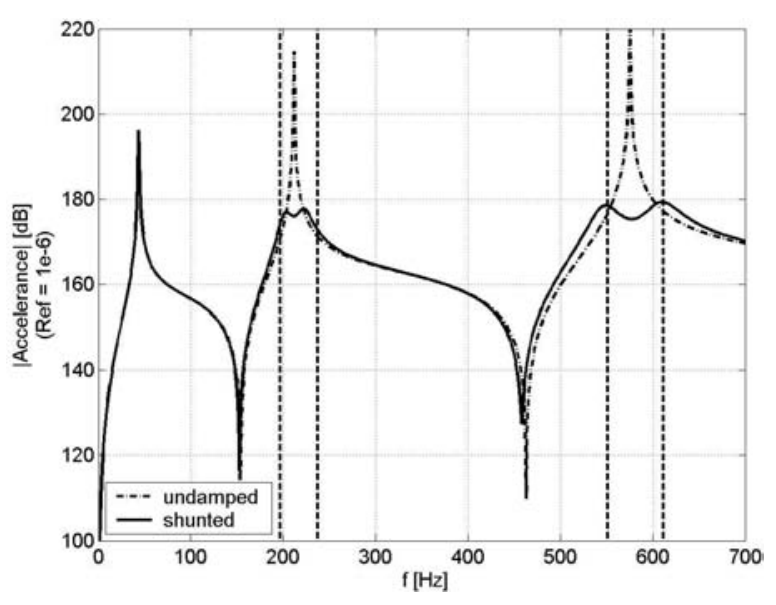

Figure 25. FRF for systems with and without passive damping.

\section{Optimal Design Strategy for Multimodal Shunted Piezoelectrics}

Equation (28) shows that a modification introduced in any mode influences the FRF in the whole frequency band. This means that the effect of a resonant shunt circuit, tuned for a specific mode, does not influence only that single frequency, but the whole frequency band. Therefore, considering the design for multimodal passive vibration suppression systems, the circuits cannot be designed separately, using closed solutions, as for the single mode case. Thus, the most effective strategy should consider the influence of each mode and the band of interest. In this work, the design of the resonant shunt circuits is treated as an optimization problem, as presented in Steffen and Inman (1999) and Rade and Steffen (2000).

For illustration, consider the situation shown in Figure 24-(b) and described by Eq. (28). The response, $H_{\mathrm{ik}}(s)$, must be minimized over predefined frequency bands as chosen by the user. For example, in the case illustrated by Figure 24 and Figure 25, the second and third modes were taken as target modes for attenuation purposes. Consequently, in the most general case, the system may have a group of $N$ piezoelectric patches and their shunt circuits, each one tuned up for a different mode. Under these circumstances, the optimal design of shunt circuits consists of determining the value of each one of the $N$ inductors and $N$ resistors to be used in the shunt circuits.

Then, the optimization problem is defined as the minimization of the objective function given by:

$$
J(\{L\},\{R\})=\sum_{m=1}^{p}\left|H_{i k}\left(s_{m}\right)\right|
$$

subject to:

$$
\begin{aligned}
L_{i}^{\text {lower }}<L_{i}<L_{i}^{\text {upper }}, & \\
R_{i}^{\text {lower }}<R_{i}<R_{i}^{\text {upper }}, &
\end{aligned}
$$

where:

- $p:$ is the number of values of $H_{\mathrm{ik}}(s)$; these values are computed by using the modeling of multi-modal 
piezoelectric shunt systems as shown in the previous section,

- $H_{\mathrm{ik}}(s):$ is the frequency response function,

- $s_{m}$ : represents the Laplace variable within the frequency band of interest;

- $L_{i}$ and $R_{i}$ : are the inductors and resistors to be used in the damping of the ith mode, respectively, and

- $N$ : is the number of shunt circuits to be used.

The optimization task that defines the design of the shunt circuits is an example of direct problem. In spite of this, the use of classical optimization methods fails in many cases, due to local minima found in the design space. For this reason, in the present work, a natural optimization method was used, namely the LifeCycle Model, to be briefly reviewed in the following subsection.

\section{LifeCycle Model - an Overview}

LifeCycle Model (Krink and Løvberg, 2002) is inserted in the natural optimization context, in which Genetic Algorithms (GA) and Particle Swarm Optimization (PSO) also belong. In biology, the term refers to the passage through the phases during the life of an individual. As life phases, can be cited the sexual maturity and the mating seasons, for example. As it happens in nature, the ability of an individual to actively change its own phase or stage in response to its success in the environment is the main inspiration for LifeCycle. In fact, the idea behind LifeCycle is to use the transitions to deal with the mechanism of self-adaptation to the optimization problem. The fitness offers a criterion used by each individual to shift to another life stage. To close the definition, LifeCycle stages must be presented. In the present work, two heuristics are used as stages, namely the GA and the PSO. Other versions of the LifeCycle can be proposed by considering other heuristics and a mix of them, as shown in Krink and Løvberg (2002).

Since the algorithm is composed by various heuristics, it is necessary to set the parameters of every heuristic used in the LifeCycle model. Nevertheless, there is a parameter inherent to the LifeCycle model, namely the number of iterations that represents a stage of the LifeCycle, called as stage interval. At the end of each stage interval, the less well-succeeded individuals must change their stage in order to improve their fitness. This means that the optimization approach does not follow a rigid scheme as proposed in Assis and Steffen (2003), in which various techniques are used sequentially in a cascade-type of structure. In other words, it is the mechanism of self-adaptation to the optimization problem that counts.

It is important to notice that the algorithm can run in a parallel scheme, since the original population is divided in a subpopulation of PSO particles and another one of GA individuals. During the optimization procedure the agents of each subpopulation commute to the other in a way to improve its own fitness.

Details about GA are provided by Michalewicz (1994) and Haupt and Haupt (1998), while PSO is comprehensively presented by Kennedy and Eberhart (1995) and Venter and Sobieski (2002).

The outline of a basic LifeCycle algorithm is presented below:

1. Initialize the algorithm parameters for the PSO and GA.

2. Evaluate the fitness for all particles (PSO) and individuals (GA).If there is no recent improvement, switch the LifeCycle stage (change from GA to PSO or vice-versa).

3. For all PSO particles, run the PSO algorithm.

4. For all GA individuals, run the GA algorithm.

5. Go to step 2 and repeat until the stop criteria are achieved.
For more detailed information about LifeCycle Model the reader should refer to (Rojas et al., 2004).

\section{Experimental Results}

Using a mechanical system composed by a flexible beam, two piezoelectric patches and their respective shunt circuits, it was possible to perform the experimental verification of the design methodology presented above. Figure 26 shows details of the experimental setup. Figure 26-(a) gives the configuration used for single mode vibration suppression, in which a single piezoelectric patch and shunt circuit are used. Figure 26-(b) shows the configuration for two modes, in which a pair of PZT patches and shunt circuits is needed.

(a)

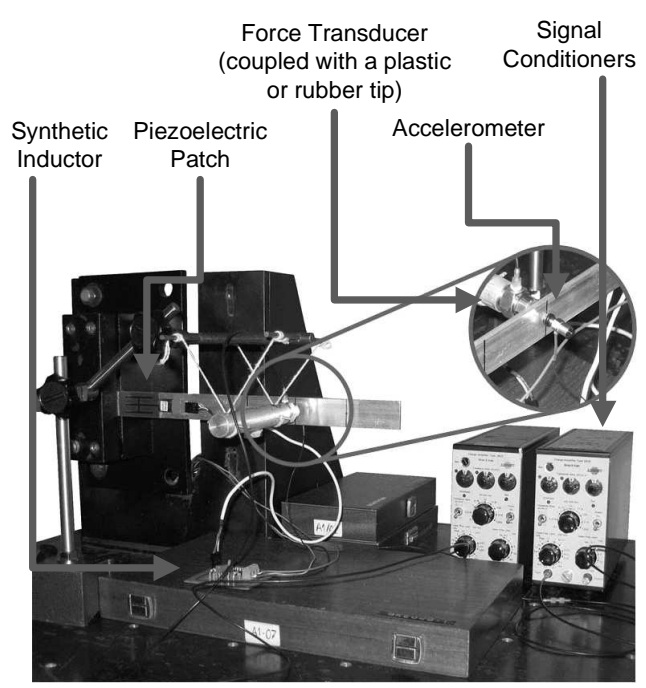

(b)

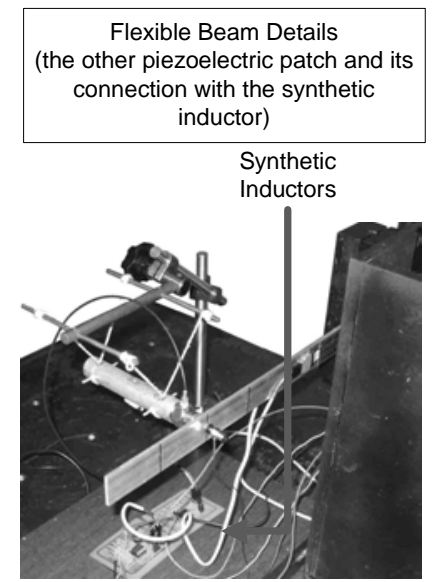

Figure 26. Experimental setup.

A pair of piezoelectric patches ACX QP10N (QP10N-11571 and QP10N-11573) was used in the experiments. As previously presented, the manufacturer furnished the information shown in Table 7. However, as was done for QP15N, the values computed in the design of the shunted PZTs were obtained from tests by using the impedance analyzer Hewlett Packard HP4194A. The corresponding experimental data are illustrated in Figure 27. 
Table 7. ACX QP10N specifications.

\begin{tabular}{|c|c|c|}
\hline Device size [mm] & Device weight[g] & $\begin{array}{c}\text { Number of } \\
\text { elements }\end{array}$ \\
\hline $50.8 \times 25.4 \times 0.381$ & 2.834 & 1 piezoelectric patch \\
\hline $\begin{array}{c}\text { Piezoelectric patch } \\
\text { size [mm] }\end{array}$ & $\begin{array}{c}\text { Device capacitance } \\
\text { [nF] }\end{array}$ & $\begin{array}{c}\text { Operation range } \\
{[\text { [V] }}\end{array}$ \\
\hline $\begin{array}{c}45.974 \times 20.574 \times \\
0.254\end{array}$ & 60 & \pm 200 \\
\hline
\end{tabular}

(a) QP10N-11571-Impedance
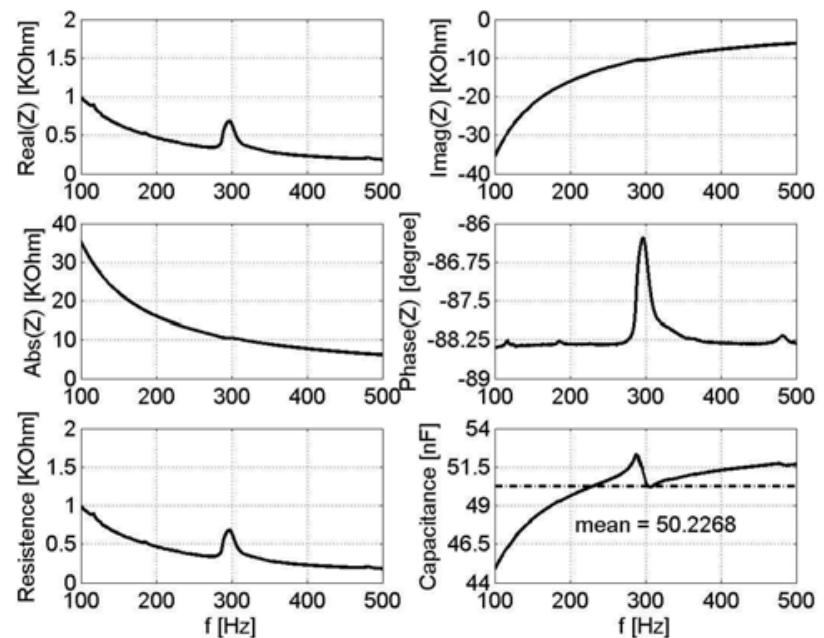

(b) QP10N-11573-Impedance
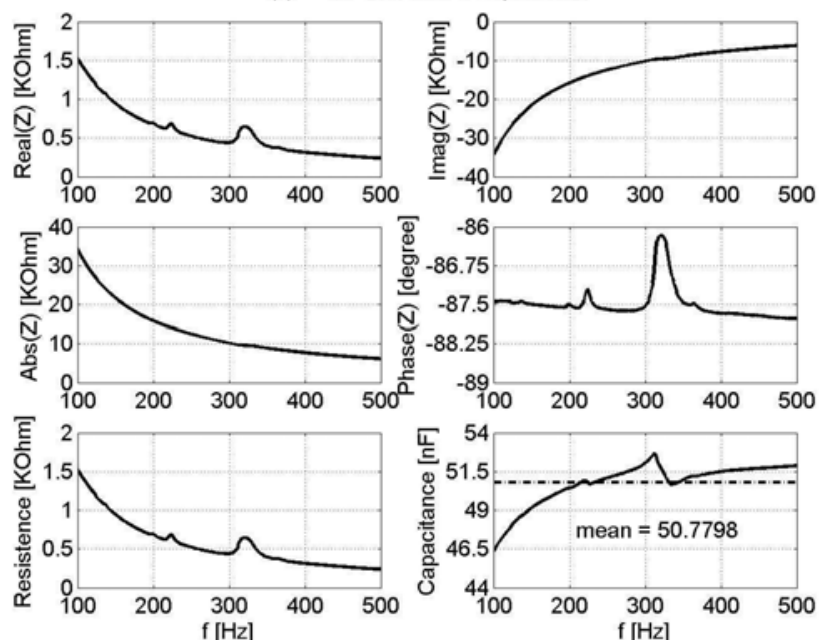

Figure 27. Impedance for the ACX QP10N + Beam system.

In the design of the shunt circuits, it was considered that the capacitance of the piezoelectric patches that should be tuned by the inductor is $C_{P Z T}=50.5033 \mathrm{nF}$ (corresponding to the average value of the capacitances shown in Figure 27).

\section{Computational Model for the Mechatronic System}

As stated above, the modal mass and modal stiffness of the system are required in the design of the piezoelectric passive damping device. For this aim, it was used the finite element method (FEM).

Figure 28 shows the FEM model of the structure: Figure 28-(a) presents a schematic representation of the system; Figure 28-(b) shows details regarding the FEM model; and Figure 28-(c) presents the degrees of freedom considered.

(a)

$3.81 \times 10^{-4} \mathrm{~m}$ thick

piezoceramics on top and

$5.1 \times 10^{-2} \mathrm{~m}$ bottom surfaces of the beam
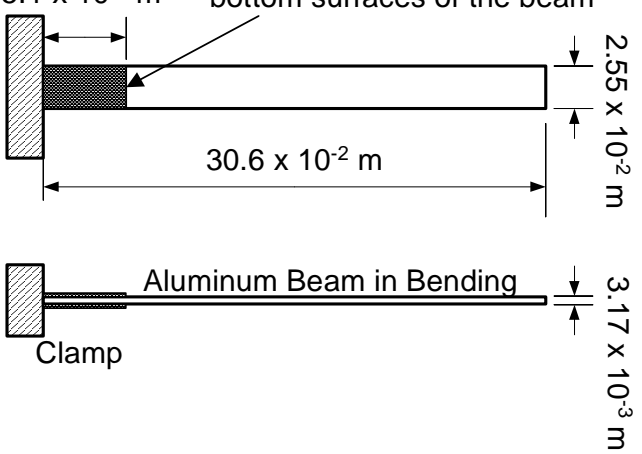

(b) Elements and nodes of the FEM Model

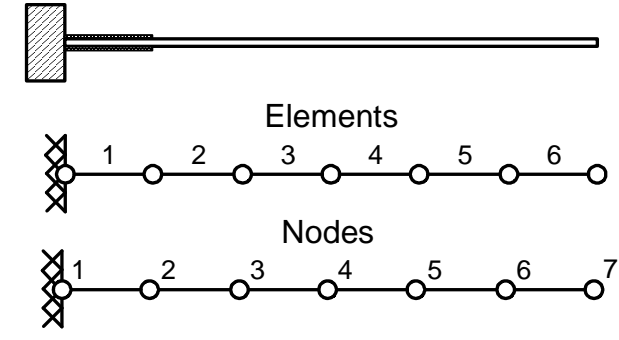

(c) DOF of the Nth Element

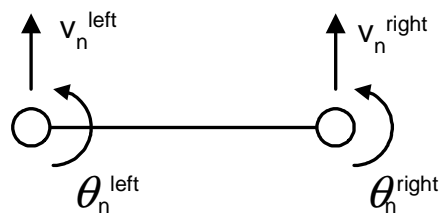

Figure 28. Mechatronic system model.

Table 8 shows the physic and geometric properties used in the FEM model.

Table 8. Properties used in the FEM model.

\begin{tabular}{|l|c|c|c|}
\cline { 2 - 4 } \multicolumn{1}{c|}{} & $\begin{array}{c}\text { Piezoelectric } \\
\text { Patch }\end{array}$ & Beam & $\begin{array}{c}\text { Nth } \\
\text { Element }\end{array}$ \\
\hline Elastic modulus [Pa] & $69 \times 10^{9}$ & $70 \times 10^{9}$ & - \\
\hline Density $\left[\mathbf{K g} / \mathbf{m}^{3}\right]$ & 7700 & 250 & - \\
\hline Length [m] & 0.051 & 0.306 & 0.051 \\
\hline Width [m] & 0.0254 & 0.0254 & 0.0254 \\
\hline Thickness [m] & 0.00381 & 0.00317 & 0.00381 \\
\hline
\end{tabular}

The classical Euler-Bernoulli theory for modeling the beam was used. To validate the model, experimental tests were performed. Figure 29 illustrates the amplitudes of the FRF obtained through the computational and experimental models. It is possible to notice an agreement in the resonance frequencies. This means that it is possible to use the model above to design the parameters of the shunt circuits. 


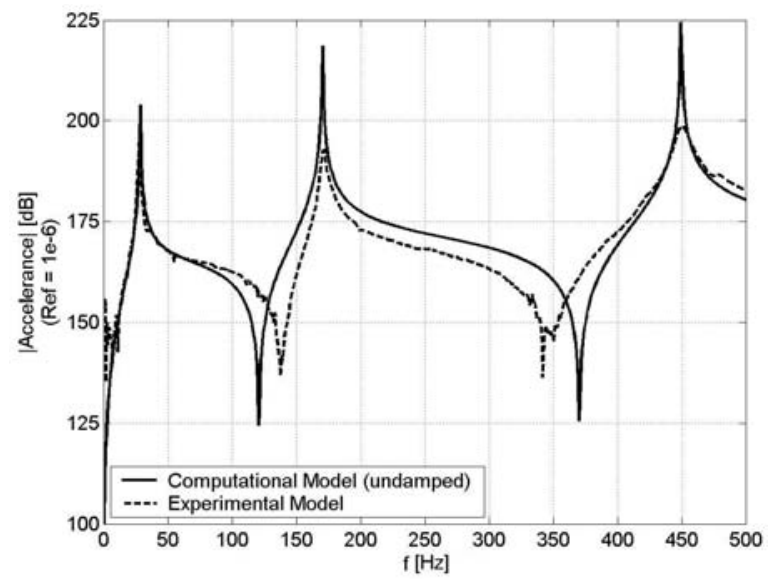

Figure 29. Computational and experimental results.

To close the requirements for the design of the shunt circuits, Table 9 shows the generalized electromechanical coupling coefficients for the first three modes of the structure.

Table 9. Generalized electromechanical coupling coefficients for each piezoelectric patch.

\begin{tabular}{|c|c|c|}
\cline { 2 - 3 } \multicolumn{1}{c|}{} & \multicolumn{2}{c|}{$\begin{array}{c}\text { Generalized Electromechanical } \\
\text { Coupling Coefficient }\end{array}$} \\
\hline Mode & QP10N-11571 & QP10N -11573 \\
\hline $\mathbf{1}^{\text {st }}$ & 0.0664 & 0.0929 \\
\hline $\mathbf{2}^{\text {nd }}$ & 0.0223 & 0.0130 \\
\hline $\mathbf{3}^{\text {rd }}$ & 0.1175 & 0.1364 \\
\hline
\end{tabular}

\section{Single Mode Case}

Considering that the capacitance of the piezoelectric patch is $C_{P Z T}=50.5033 \mathrm{nF}$ and by choosing arbitrarily the QP10N-11571 PZT patch, the optimal parameters of the resonant shunt circuits for the first three vibration modes are presented in Tab. 10.

Table 10. Optimal shunt parameters for the single mode case.

\begin{tabular}{|c|c|c|c|c|}
\cline { 2 - 5 } \multicolumn{1}{c|}{} & \multicolumn{3}{c|}{ Shunt Circuit Configuration } \\
\cline { 2 - 5 } \multicolumn{1}{c|}{ Series } & \multicolumn{2}{c|}{ Parallel } \\
\hline $\begin{array}{c}\text { Target } \\
\text { Mode }\end{array}$ & $R_{O P S M}^{R S P_{\text {SERES }}}$ & $L_{O P T M}^{R S P_{\text {SERIES }}}$ & $R_{O P T M}^{R S P_{P A R A L E L}}$ & $L_{O P T P_{P A R A L E L}}^{R T}$ \\
\hline $\mathbf{1}^{\text {st }}$ & 10.3053 & {$[\mathrm{H}]$} & {$[\mathrm{K} \Omega]$} & {$[\mathrm{H}]$} \\
\hline $\mathbf{2}^{\text {nd }}$ & 0.5837 & 17.0547 & 1174.0849 & 615.1039 \\
\hline $\mathbf{3}^{\text {rd }}$ & 1.1489 & 2.4476 & 585.416 & 17.2663 \\
\hline
\end{tabular}

As can be observed, the values of the inductors are extremely large, especially for the first mode. As previously announced in this paper, synthetic inductors are designed to overcome the problem of large inductance values. For the resistors, it is noticed that the parallel configuration leads to larger values than the series one. This means an advantage of the parallel topology as compared to the series one. Since synthetic inductor presents a parasite resistance, it creates a voltage divisor with the load resistor of the shunt circuit. Thus, in order to facilitate electric energy dissipation in the resistor designed for the shunt circuit, a smaller influence of the parasite resistance is necessary. This means that the parallel topology becomes more attractive than the series one. Consequently, in all the experiments presented in this work, it was used the parallel topology.
Figure 30 shows the results of the experiments related to the vibration reduction of a single mode. For each mode considered, the response of the shunted PZT system is compared with the open circuit case.
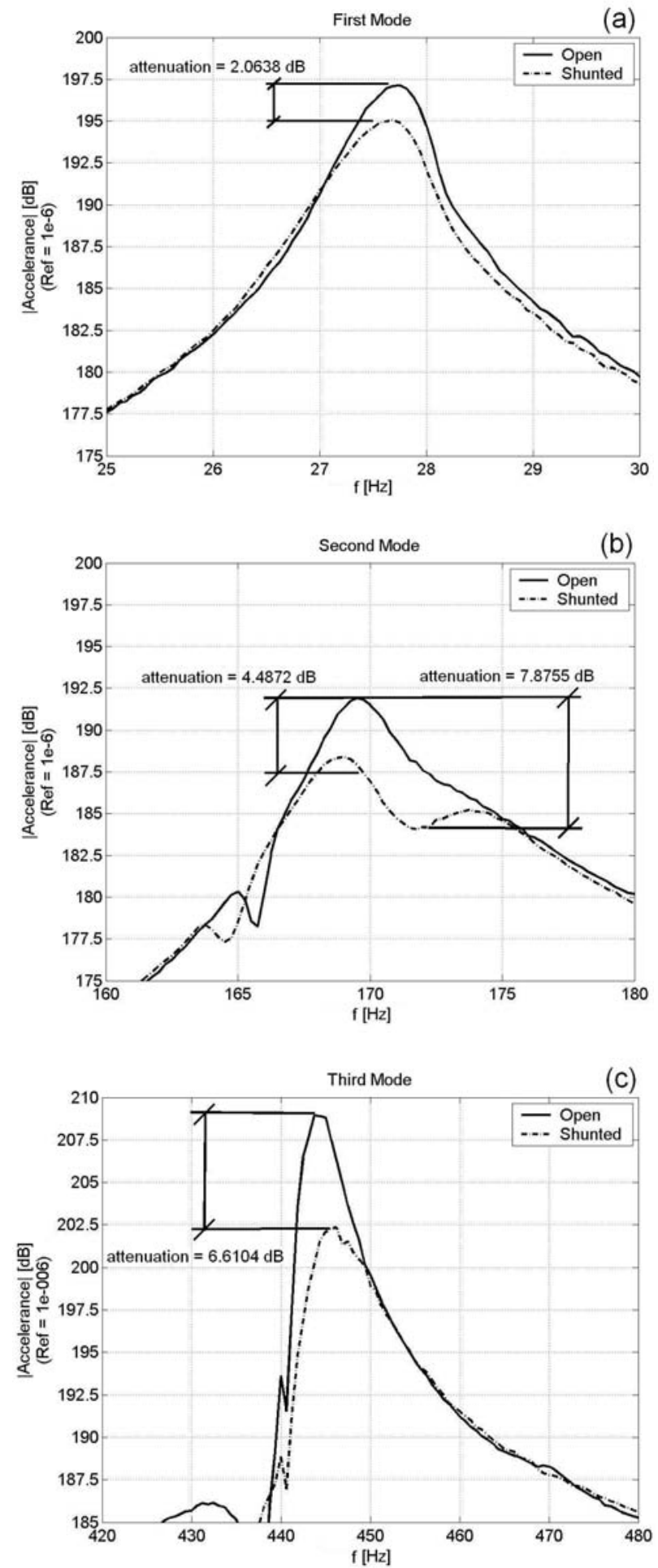

Figure 30. Experimental results for the single mode case. 


\section{Multiple Mode Case}

As described previously, the design of shunt circuit parameters for the multimode case is treated as an optimization problem. The attenuation of the vibration in the neighborhood of the second and third modes is addressed in the present work.

Table 11 gives the shunt circuit parameters as obtained by following the optimization strategy. Figure 31 gives the characteristics of the optimal design: Figure 31-(a) shows the frequency bands of the spectrum chosen to have their vibration amplitudes minimized; Figure 31-(b) and (c) give the evolution of LifeCycle along the optimization process.

Table 11. Optimal design of the shunt circuit parameters for the $1^{\text {st }}$ and $2^{\text {nd }}$ modes.

\begin{tabular}{|c|c|c|c|}
\hline Mode & Patch & $R_{O P T M}[\mathrm{~K} \Omega]$ & $L_{O P T M}[\mathrm{H}]$ \\
\hline $1^{\text {st }}$ & QP10N-11571 & 1125.8039 & 606.7015 \\
\hline $2^{\text {nd }}$ & QP10N-11573 & 821.2278 & 17.1836 \\
\hline
\end{tabular}
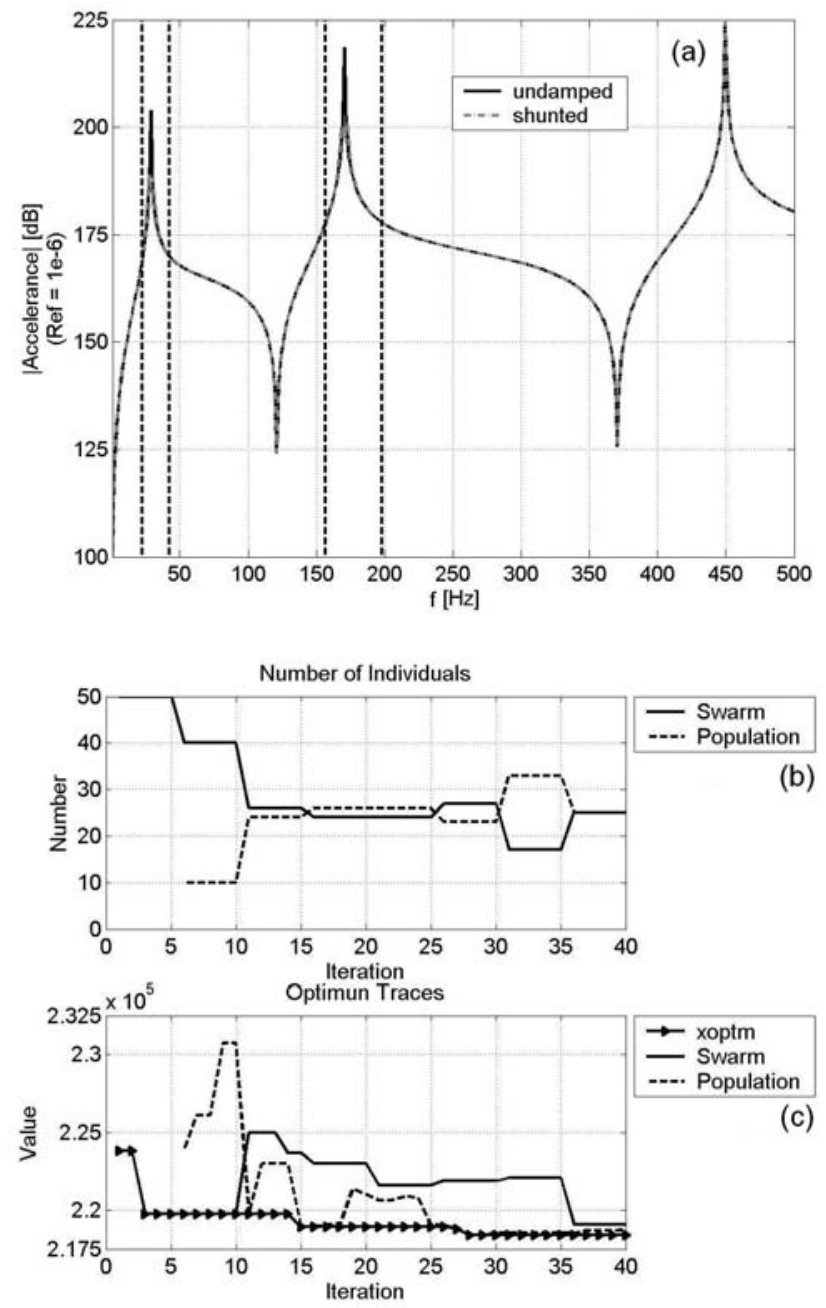

Figure 31. Optimization for $\mathbf{1}^{\text {st }}$ and $2^{\text {nd }}$ modes - multimode case.

It is important to point out the importance of the optimization process in the design of the parameters of the shunt circuits. In one hand, the parameters calculated by using the single mode formulation give the final value of the objective function equal to $J\left(\left\{\mathbf{L}_{\text {single }}\right\},\left\{\mathbf{R}_{\text {single }}\right\}\right)=2.2085 \times 10^{5}$. On the other hand, the design obtained from the optimization process gives $J\left(\left\{\mathbf{L}_{\text {optm }}\right\},\left\{\mathbf{R}_{\text {optm }}\right\}\right)=2.1842 \times 10^{5}$.

Figure 32 shows the experimental results obtained for the multimode case.
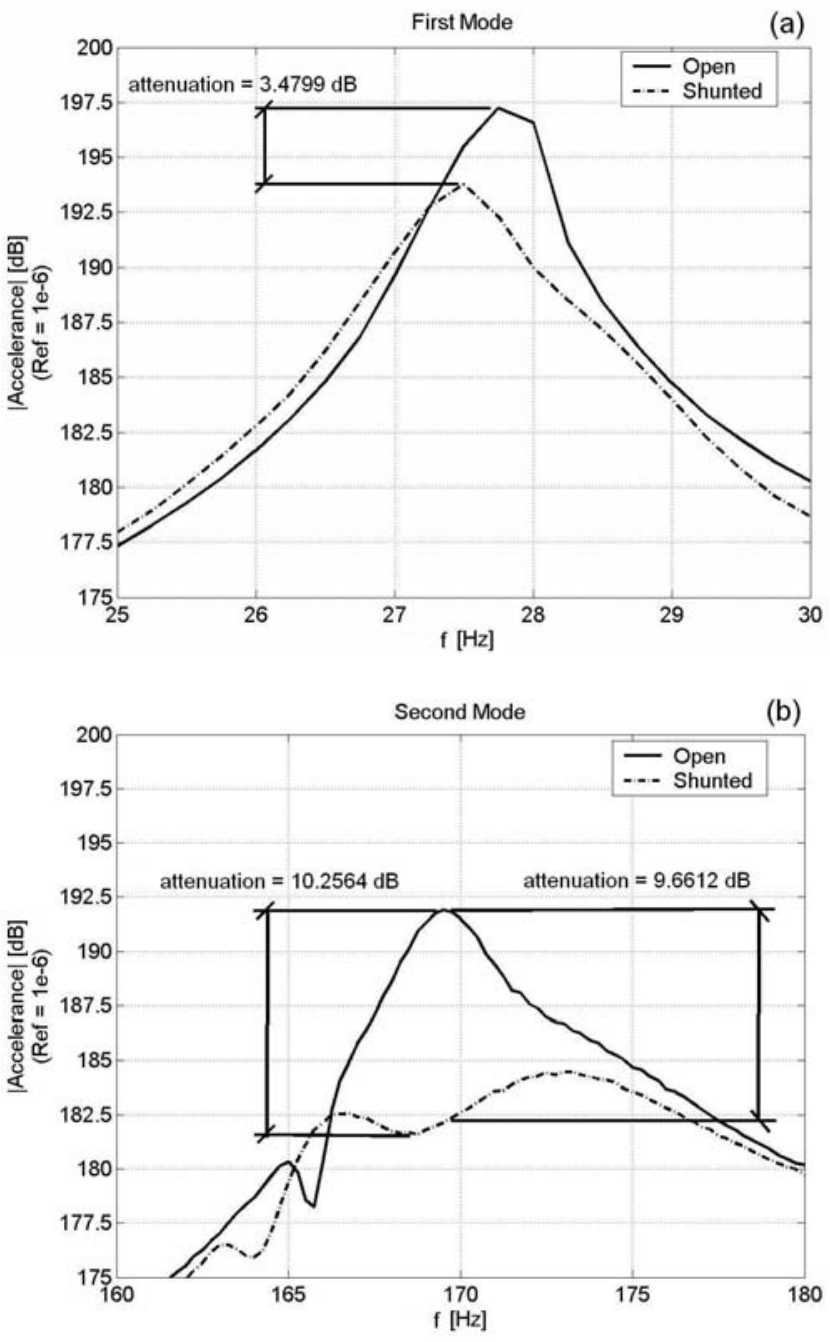

Figure 32. Experimental results for the $1^{\text {st }}$ and $2^{\text {nd }}$ modes - multimode case.

Table 12 presents the shunt circuit parameters, as obtained by using the optimization approach. Figure 33 shows the characteristics of the optimal design: Figure 33-(a) exhibits the frequency bands of the spectrum chosen to have their vibration amplitudes minimized; Fig. 33-(b) and (c) give the evolution of LifeCycle along the optimization process.

Table 12. Optimal design of the shunt circuit parameters for the $2^{\text {nd }}$ and $3^{\text {rd }}$ modes - multimode case.

\begin{tabular}{|c|c|c|c|}
\hline Mode & Patch & $R_{\text {OPTM }}[\mathrm{K} \Omega]$ & $L_{O P T M}[\mathrm{H}]$ \\
\hline $2^{\text {nd }}$ & QP10N-11571 & 814.8445 & 17.1468 \\
\hline $3^{\text {rd }}$ & QP10N-11573 & 53.7718 & 2.5338 \\
\hline
\end{tabular}



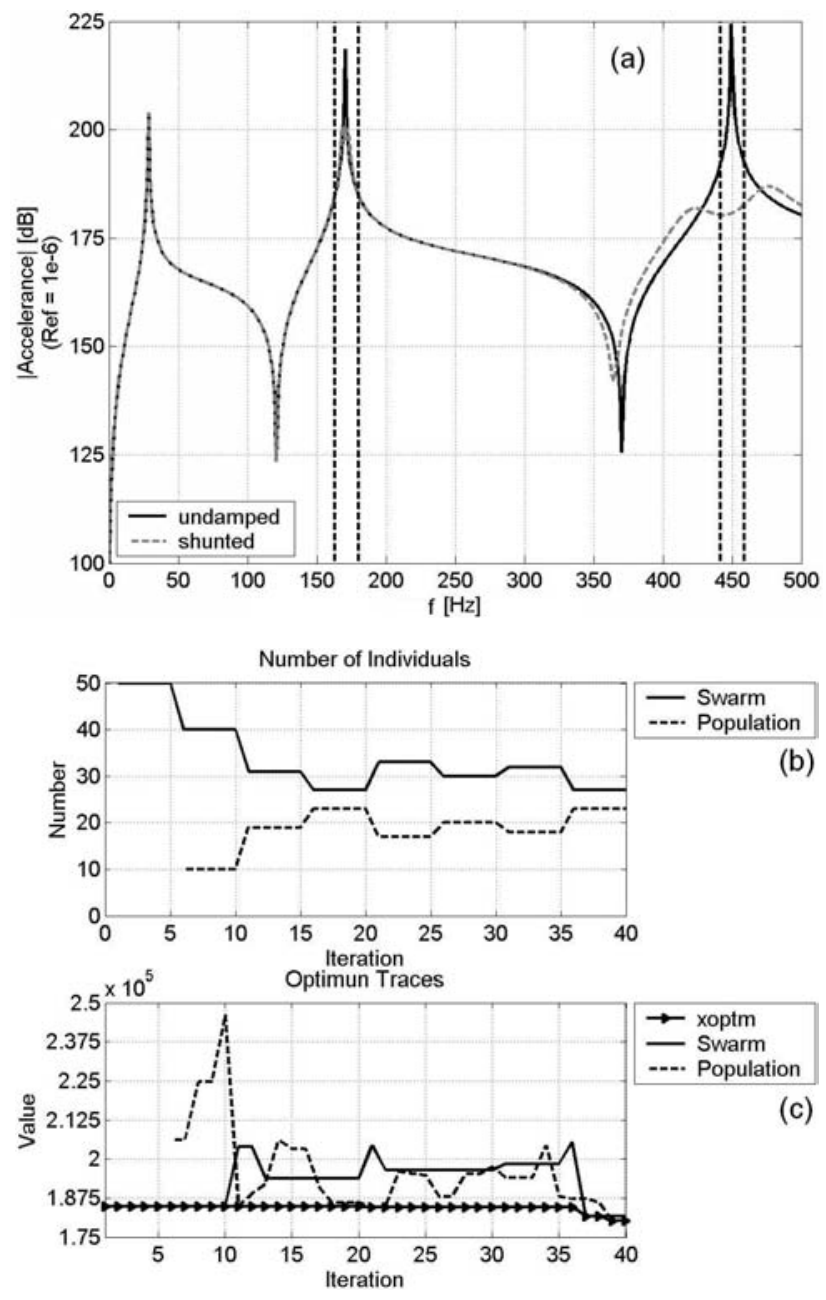

Figure 33. Optimization for $2^{\text {nd }}$ and $3^{\text {rd }}$ modes - multimode case.

It is worth pointing out the importance of the optimization process in the design of the parameter of the shunt circuits when various modes are considered simultaneously. The parameters calculated by using the single mode formulation lead to the final value of the objective function equal to $J\left(\left\{\mathbf{L}_{\text {single }}\right\},\left\{\mathbf{R}_{\text {single }}\right\}\right)=1.9407 \times 10^{5}$. However, the design obtained from the optimization process (multimode case) gives $J\left(\left\{\mathbf{L}_{\mathrm{optm}}\right\},\left\{\mathbf{R}_{\mathrm{optm}}\right\}\right)=1.7812 \times 10^{5}$.

Figure 34 shows the experimental results for the second and third modes, obtained for the multimode case.

It is worth mentioning that the results concerning the multimode case take into account two piezoelectric patches bonded to the structure. Each patch is tuned to a specific mode. Consequently, the amount of energy dissipated through this system is larger than the one found for the single mode case (in which a single patch was used).
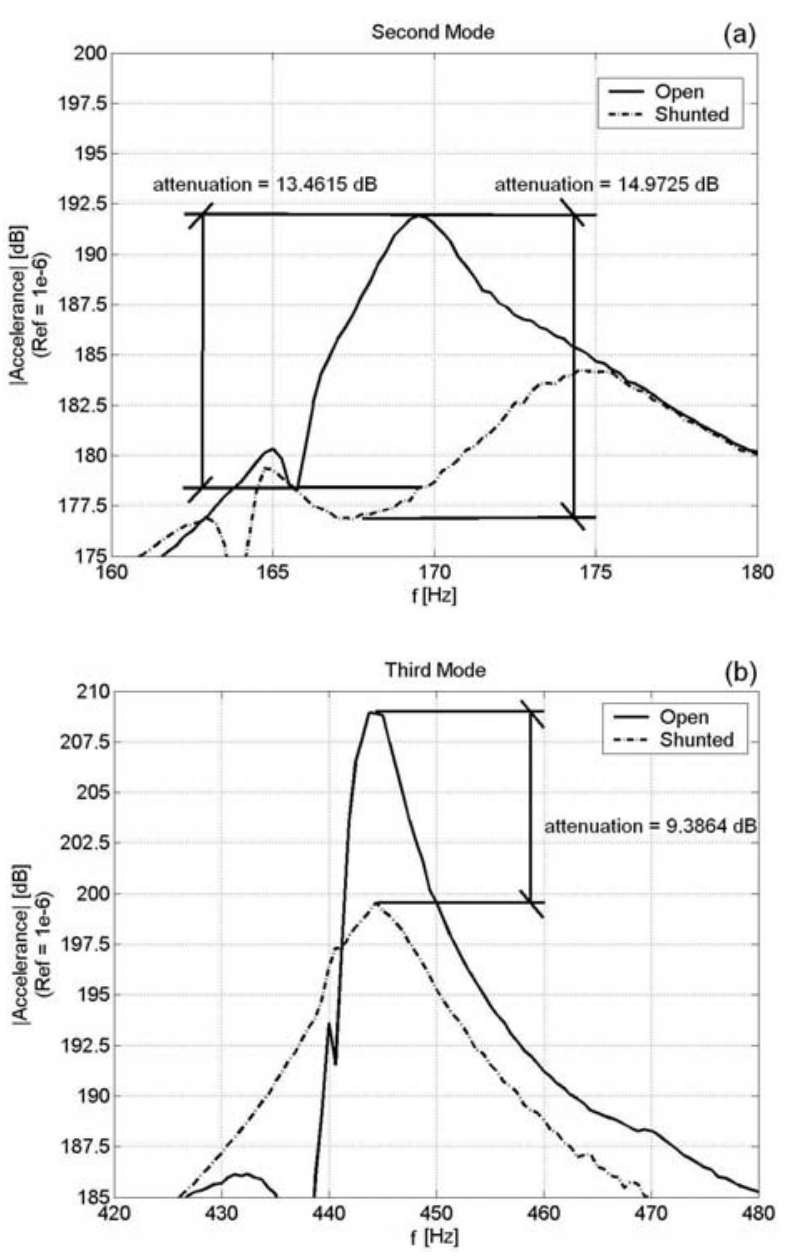

Figure 34. Experimental results for the $2^{\text {nd }}$ and $3^{\text {rd }}$ modes - multimode case.

\section{Conclusions}

The present contribution was dedicated to the study of passive vibration damping by using piezoelectric patches and resonant shunt circuits. The analytical model of the system shows that the general behavior of shunted piezoelectric systems is similar to the classical dynamic vibration absorber. This paper presented a complete study concerning resonant shunted piezoelectric, including the analytical and experimental aspects together with the synthetic inductor design. This was intended to offer a complete study regarding resonant shunted piezoelectric, allowing further implementations by the interested reader. Besides, the study devoted to the multimode case as developed by the authors represents a more realistic approach. It should be considered that the multimode case is not a simple extension of the single mode case. Also, the use of optimization techniques can be considered a valuable contribution because no closed solution can be derived for the multimode case. The optimization results were validated experimentally. It was observed that it is possible to obtain closed form solutions for the shunt parameters only in the case of single mode attenuation. An important aspect is that the multimode technique is mandatory in a great number of real situations, in which the modes are quite close in the frequency spectrum. In such cases the influence of each mode in the response cannot be discarded. It was necessary to design synthetic inductors based on operational amplifiers, to avoid cumbersome traditional inductors. Details were given about the 
design of synthetic inductors and experimental tests were included to help in the choice of the circuits' parameters. Both numerical and experimental results are very encouraging in the sense that electric shunt circuits can be successfully used in order to attenuate vibrations of flexible structures. Further studies will be focused on other attenuation schemes such as the one proposed by the so-called negative impedance converter.

\section{Acknowledgment}

The authors are thankful to CNPq Brazilian Research Agency for the financial support for the present research work through Proc.Nb. 470082/2003-8. The authors are also grateful to EMBRACO for their interest and support.

\section{References}

Akhras, G., 2000, "Smart Materials and Smart Systems for the Future", Canadian Military Journal, Vol.1 N 3 , pp 24-31.

Antoniou, A., 1969, "Realization of Gyrators using Operational Amplifiers, and their use in RC-Active-Network Synthesis", IEEE Proc., Vol. 116, No 11 , pp. 1838-1850.

Assis, E.G. e Steffen Jr, V., 2003,'Inverse Problem Techniques for the Identification of Rotor-Bearing Systems", Inverse Problems in Engineering, Vol. 11, No. 1, Taylor \& Francis, pp. 39-53.

Caruso, G., 2001, "A Critical Analysis of Electric Shunt Circuits Employed in Piezoelectric Passive Vibration Damping", Smart Materials and Structures Vol. 10, pp. 1059-1068.

Fleming, A. J., Behrens, S. e Moheimani, S. O. R., 2002, "Optimization and Implementation of Multimode Piezoelectric Shunt Damping Systems", IEEE/ASME Transactions on Mechatronics, Vol. 7, $\mathrm{N}^{\circ}$ 1, pp. 87-94.

Fleming, A. J., Behrens, S. e Moheimani, S. O. R., 2003, "Reducing the Inductance Requirements of Piezoelectric Shunt Damping Systems", Smart Materials and Structures Vol. 12, pp. 57-64.

Hagood, N. W. e von Flotow, A., 1991, "Damping of Structural Vibrations with Piezoelectric Materials and Passive Electrical Networks", Journal of Sound and Vibration, Vol. 146, №2, pp 243-268.

Haupt, R. L. and Haupt, S. E., 1998, "Practical Genetic Algorithms", Wiley-Interscience Publication, New York.

Johnson, C. D., 1995, "Design of Passive Damping Systems", Journal of Vibration and Acoustics Vol. 117, Special 50th Anniversary Design Issue, pp 171-176.
Kennedy, J. and Eberhart, R. C., 1995, "Particle Swarm Optimization", Proceedings of the 1995 IEEE International Conference on Neural Networks, Perth, Australia, pp. 1942-1948.

Krink, T. and Løvberg, M., 2002, "The LifeCycle Model: Combining Particle Swarm Optimisation, Genetic Algorithms and HillClimbers", Proceedings of the 7th International Conference on Parallel Problem Solving from Nature, Granada, Spain, pp. 621-630.

Lesieutre, G. A., 1998, "Vibration Damping and Control using Shunted Piezoelectric Materials", Shock and Vibration Digest, Vol. 30, pp 181-190.

Maia, N. M. M. and Silva, J. M. M., 1997, “Theorical and Experimental Modal Analysis”, Research Studies press LTD.

Massara, R.E., Steadman, J.W., Wilamowski, B.M. and Svoboda, J.A., 2000, "The Electrical Engineering Handbook", Ed. Richard C. Dorf Boca Raton: CRC Press $L L C$

Michalewicz, Z., 1994, "Genetic Algorithms + Data Structures = Evolution Programs", $2^{\text {a }}$ edition, Springer-Verlag, New York.

Park, C. H. and Inman, D. J., 2003, "Enhanced Piezoelectric Shunt Design", Shock and Vibration, Vol. 10, No. 2, pp 127-133.

Pereira, C.B., 2003, "Discrete-Continuous Optimization Techniques Applied to Vibration Control of Intelligent Structures", MSc Theses (in portuguese), School of Mechanical Engineering, Federal University of Uberlândia.

Rade, D. A. and Steffen Jr., V., 2000, "Optimisation of Dynamic Vibration Absorvers over a Frequancy Band", Mechanical Systems and Signal Processing 14, 679-690.

Riordan, R. H. S., 1967, "Simulated inductors using differential amplifiers", Electronic Letters, Vol. 3, pp 50-51.

Rojas, J.E.F., Viana, F.A.C., Rade, D.A. and Steffen Jr, V., 2004, "Identification of external loads in mechanical systems through heuristicbased optimization methods and dynamic responses", Latin American Journal of Solids and Structures; Vol. 1, No. 3, pp. 297-318.

Steffen Jr., V. and Inman, D. J., 1999, "Optimal Design of Piezoelectric Materials for Vibration Damping in Mechanical Systems", Journal of Intelligent Material Systems and Structures, Vol. 10, pp 945-955.

Steffen Jr, V., Rade, D. A. and Inman, D. J., 2000, "Using Passive Techniques for Vibration Damping in Mechanical Systems"; Journal of the Brazilian Society of Mechanical Sciences, Vol XXII, No. 3, pp. 411-421.

Venter, G. and Sobieszczanski-Sobieski, J., 2002, "Particle Swarm Optimization", Proceedings of the 43rd AIAA/ASME/ASCE/AHS/ASC Structures, Structural Dynamics, and Materials Conference, Vol. AIAA2002-1235.

Viana, F. A. C., 2005, "Vibration Damping by using Piezoelectric Patches and Resonant Shunt Circuits" (in Portuguese), M. Sc. Dissertation, Federal University of Uberlândia, $\mathrm{MG}$ - Brazil.

Wu, S. Y., 1996, "Piezoelectric Shunts with Parallel R-L Circuit for Structural Damping and Vibration Control", Proc. SPIE Smart Structures and Materials, Passive Damping and Isolation; SPIE Vol. 2720, pp 259-269 